University of Louisville

ThinkIR: The University of Louisville's Institutional Repository

Electronic Theses and Dissertations

$12-2011$

\title{
Rapid functionalization of gold monolayer protected clusters for fabricating electronic noses.
}

Yang Yang

University of Louisville

Follow this and additional works at: https://ir.library.louisville.edu/etd

\section{Recommended Citation}

Yang, Yang, "Rapid functionalization of gold monolayer protected clusters for fabricating electronic noses." (2011). Electronic Theses and Dissertations. Paper 1616.

https://doi.org/10.18297/etd/1616

This Master's Thesis is brought to you for free and open access by ThinkIR: The University of Louisville's Institutional Repository. It has been accepted for inclusion in Electronic Theses and Dissertations by an authorized administrator of ThinkIR: The University of Louisville's Institutional Repository. This title appears here courtesy of the author, who has retained all other copyrights. For more information, please contact thinkir@louisville.edu. 


\title{
RAPID FUNCTIONALIZATION OF GOLD MONOLAYER PROTECTED CLUSTERS FOR FABRICATING ELECTRONIC NOSES
}

\author{
By \\ Yang Yang

\begin{abstract}
A Thesis
Submitted to the Faculty of the

Graduate School of the University of Louisville

in Partial Fulfillment of the Requirements

for the Degree of
\end{abstract} \\ Master of Science \\ Department of Chemistry \\ University of Louisville \\ Louisville, Kentucky
}

December 2011 


\title{
RAPID FUNCTIONALIZATION OF GOLD MONOLAYER PROTECTED CLUSTERS FOR FABRICATING ELECTRONIC NOSES
}

\author{
by \\ Yang Yang \\ A Thesis Approved on \\ December 09, 2011 \\ by the following Thesis Committee:
}

Thesis Director Dr. Francis P. Zamborini

Dr. Xiang Źhang

Dr. Gamini Sumanasekera 


\section{DEDICATION}

This thesis is dedicated to my parents

Jianyuan Yang and Meiqing Zhou

who gave me invaluable educational opportunities. 


\section{ACKNOWLEDGEMENTS}

Here I would like to acknowledge the people that helped me during these years to complete my degree. First, I would like to thank my advisor, Dr. Francis P. Zamborini for his excellent guidance and constant support during my research. He helped me in many steps of my work and always believed in me. I am very grateful I could be his student.

I would like to thank my research group members, Monica Moreno, Lanlan Bao, Rafael Masitas, Tulashi Luitel, Nidhi Shah, Irina Plotnikova, Aiqin Fang, Dr. Olga Ivanova, Dr. Grzegorz Slawinski, Dr. Srinivas Beeram, and Dr. Radhika Dasari for constant support and good advice.

Of course, I am grateful to my parents Jianyuan Yang and Meiqing Zhou, to whom this work is dedicated, for their patience, understanding, love and encouragement to follow my dreams. Without them this work would never have come into existence. 


\section{ABSTRACT \\ RAPID FUNCTIONALIZATION OF GOLD MONOLAYER \\ PROTECTED CLUSTERS FOR FABRICATING ELECTRONIC NOSES}

Yang Yang

December 9th, 2011

This thesis describes 1) the kinetics of vapor phase place exchange reactions on films of gold monolayer protected clusters (Au MPCs) to alter the functionality of the $\mathrm{Au}$ MPCs, 2) applications of the reaction for altering chemiresistive sensing selectivity for volatile organic compounds (VOCs), and 3) the fabrication of a chemiresistive electronic nose for detecting VOCs based on sensor arrays and vapor phase place-exchange reaction. The motivation of this work was to develop a simple and effective method to synthesize and functionalize films of $\mathrm{Au}$ MPCs for applications in nanoelectronics, sensing, and catalysis.

We first synthesize hexanethiolate-coated gold monolayer-protected clusters (C6S Au MPCs) and then prepare a film by drop-cast deposition. Placing the film of C6S Au MPCs into a closed container with mercaptoethanol leads to the replacement of the hexanethiolate ligands with mercaptoethanol ligands by vapor phase 
place-exchange. Nuclear magnetic resonance (NMR) and Fourier transform infrared (FTIR) spectroscopy allowed a determination of the reaction kinetics. After one day of exchange, up to 91 percent of the hexanethiolates (C6S) were replaced with mercaptoethanol (OHC2S). The resistance of the films decreased from $250 \mathrm{M} \Omega$ to $150 \mathrm{M} \Omega$ after the one-day exchange. Importantly, the chemiresistive sensing response ratio to isopropanol (IPA) versus toluene gradually changed from 0.3 (IPA/TOL) for pure C6S Au MPCs to 1.8 after exchange with mercaptoethanol (91\%).

In addition to mercaptoethanol, we exchanged films of C6S Au MPCs with mercaptopropionic acid (MPA) and mercaptopropyltrimethoxy silane (MPTMS) for building a sensor array consisting of four devices to detect various VOCs, including toluene, IPA, ethanol, and acetone with improved selectivity and recognition. These electronic nose devices, created through vapor phase place exchange reactions, generate a unique response pattern for each of the vapors, allowing us to differentiate them by pattern recognition methods. This study Indicates that vapor phase place exchange reactions have broad potential applications in selective vapor sensing.

The vapor phase place exchange reaction can be used for mass production of films of metal MPCs with various functionalities. There are several benefits over solution phase place exchange or direct synthesis of Au MPCs with different functionality, such as it is simple, fast, massively parallel, allows functionalities that would not be possible in solution, and provides control over the extent of exchange from 0 to almost $100 \%$. Potential future applications include synthesizing metal MPC catalysts, electronic sensors, and chemical separators easily with low cost. 


\section{TABLE OF CONTENTS}

DEDICATION

PAGE

ACKNOWLEDGEMENTS

iii

ABSTRACT

iv

LIST OF TABLES

$\mathrm{V}$

LIST OF FIGURES

ix

$\mathrm{X}$

\section{CHAPTER}

I. INTRODUCTION

1.1. Main Goal/Summary 1

1.2. Motivation/Objective 1

1.3. Synthesis and Functionalizaiton of Au Monolayer Protected Clusters 2

1.3.1. Citrate reduction method 3

1.3.2. Brust-Schiffrin method 5

$\begin{array}{ll}\text { 1.3.3. Place exchange reactions } & 7\end{array}$

1.4. Electronic Properties of Au MPC Films 9

1.5. Chemiresistive Sensing $\quad 12$

1.6. Electronic Noses $\quad 15$

$\begin{array}{ll}\text { 1.7. Summary } & 18\end{array}$

II. EXPERIMENTAL 20

2.1. Synthesis of Hexanethiol-Coated Au Monolayer

Protected Clusters (MPCs) 20

2.2. Au IDA Electrode Device Fabrication and Drop Cast Deposition 21

2.3. Vapor Phase Place Exchange 23

2.4. Chemiresistive Vapor Sensing Set Up 25

2.5. Spectroscopy Measurements 28

$\begin{array}{ll}\text { 2.3.1. FTIR measurements } & 28\end{array}$

$\begin{array}{ll}\text { 2.3.2. NMR measurements } & 28\end{array}$

III. VAPOR PHASE THIOL-PLACE EXCHANGE REACTIONS

ON FILM OF GOLD MONOLA YER-PROTECTED CLUSTERS

FOR SELECTIVE SENSING OF VOLATILE ORGANIC

COMPOUNDS

30

3.1. Introduction 31 
3.2. Experimental $\quad 33$

3.2.1. Chemicals 33

3.2.2. Synthesis 33

3.2.3. Vapor exchange setup $\quad 33$

3.2.4. Vapor sensing experiment 33

3.3. Results and Discussion $\quad 35$

3.3.1. Vapor phase place-exchange reaction kinetics 35

3.3.2. Chemiresistive sensing response towards IPA

3.4. Conclusions 45

IV. CREATING SENSOR ARRAYS FOR VOLATILE ORGANIC COMPOUNDS USING VAPOR PHASE PLACE EXCHANGE ON GOLD MONOLAYER-PROTECTED CLUSTERS 46

4.1. Introduction 46

4.2. Experimental $\quad 48$

4.2.1. Chemicals 48

4.2.2. Synthesis $\quad 49$

4.2.3. Vapor exchange setup 49

4.2.4. Vapor sensing experiment 49

4.3. Results 49

4.3.1. Vapor Phase Place-Exchange Reactions for MPA and MPTMS on C6S Au MPCs 49

4.3.2. Chemiresistive Vapor Sensing and Response Patten Generated by Electronic Nose 52

4.3.3 Response Pattern Generated by Electronic Nose $\quad 59$

4.4. Conclusions $\quad 59$

V. SUMMARY AND FUTURE DIRECTIONS 63

REFERENCES 66

$\begin{array}{ll}\text { CURRICULUM VITAE } & 74\end{array}$ 


\section{LIST OF TABLES}

TABLE

PAGE

4.1. Chemiresistive sensing response to VOCs (toluene, IPA, ethanol, acetone) generated by sensors with films of $\mathrm{Au}$ MPCs coated with C6S, C6S/OHC2S (78\%OHC2S), C6S/MPA (85\%MPA), and C6S/MPTMS (87\%MPTMS) Au MPCs 


\section{LIST OF FIGURES}

FIGURE

PAGE

1.1. Procedure for synthesizing electrostatically-stabilized Au NPs by the citrate reduction reaction.(adapted from reference ${ }^{9}$ )

1.2. Procedure for synthesizing thiol-coated Au MPCs through the Brust reaction. (Adapted from reference ${ }^{10}$ )

1.3. General scheme for the place exchange reaction between

$\mathrm{Au}$ MPCs and various functionalized thiols.

$\mathrm{R}=\mathrm{Br}, \mathrm{CN}, \mathrm{Ph}, \mathrm{OH}, \mathrm{COOH}, \mathrm{F}, \mathrm{Si}\left(\mathrm{OCH}_{3}\right)_{3}$, etc.

(adopted from reference ${ }^{19}$ )

1.4. Scheme for electron hopping mechanism. Electron hops over between the metal cores when potential is applied. $\delta_{\mathrm{e}}$ is the separating distance between metal cores. (adopted from reference ${ }^{28}$ )

1.5. Scheme of a chemiresistive sensor. $A_{V}$ : analytes in environment $A_{F}$ : analytes in chemiresistor. When the device is exposed to the analytes, the interaction between the chemiresistive transducer and the analyte changes the conductivity of the chemiresistor and generate a current signal. (adopted from reference ${ }^{l}$ )

1.6. Scheme for the basic electronic nose instrument setup. The electronic nose systems simulate the human olfactory system, resulting in the recognition of volatile molecules by pattern recognition analysis.

(adopted from reference ${ }^{56}$ )

2.1. Figure of $\mathrm{Au}$ MPC sensor A: Blow up of $23 \mu \mathrm{m}$ gap region of the IDA electrode. B: C6S Au MPC film drop cast deposited on the IDA electrode. (adapted from reference ${ }^{62}$ ) 
2.2. Scheme of the experimental set up for the vapor phase place exchange reactions and the thiols we used as exchange reagents.

2.3. The chemiresistive vapor sensing set up with the film of Au MPCs in the sample chamber.

2.4. Typical chemiresistive sensing plot and response calculation.

2.5. Scheme of the FTIR and NMR measurements. A film of Au MPCs was drop-cast deposited on $\mathrm{KBr}$ salt plate and placed into the exchange device for several times and removed for the FTIR and NMR measurements.

3.1. Scheme of vapor phase exchange on film of $\mathrm{C} 6 \mathrm{~S}$ Au MPCs using OHC2S as exchange reagent. In the vapor phase, the $\mathrm{OHC} 2 \mathrm{~S}$ replaced the original $\mathrm{C} 6 \mathrm{~S}$ ligand leading to $\mathrm{C} 6 \mathrm{~S} / \mathrm{OHC} 2 \mathrm{~S}$ Au MPCs formation.

3.2. A series of FTIR spectra of OHC2S exchange for $0,1,4,24$, and $48 \mathrm{~h}$. FTIR analysis indicated that C6S ligands were gradually replaced by OHC2S ligands.

3.3. A series of NMR spectra of $\mathrm{OHC} 2 \mathrm{~S}$ exchange for $0,1,4,24$, and $48 \mathrm{~h}$. NMR analysis indicated that $\mathrm{C} 6 \mathrm{~S}$ ligands were gradually replaced by OHC2S ligands.

3.4. NMR and FTIR comparison for the $\mathrm{OHC} 2 \mathrm{~S}$ exchange ratio during the vapor phase place exchange.

3.5. FTIR samples on 2 different day. Sample $\# 1$ and $\# 2$ were performed on the same day and sample \#3 performed on a different day.

3.6. A) NMR spectra for 4 different MPC films of different thickness after a $4 \mathrm{~h}$ exchange with OHC2S: $\mathrm{S} 1$ is thinnest, $\mathrm{S} 2$ is double $\mathrm{S} 1, \mathrm{~S} 3$ is triple, and $\mathrm{S} 4$ is fourfold in film thickness. B) The OHC2S percentage after $4 \mathrm{~h}$ exchange as a funtion of relative thickness determined by NMR.

3.7. Chemiresistive sensing response of a film of C6S Au MPCs to $52 \%$ IPA and toluene after exchange for A) $0 \mathrm{~h}, \mathrm{~B}) 1 \mathrm{~h}$, and C) $24 \mathrm{~h}$. The ON and OFF arrows indicate when the sensor is exposed to the vapor (ON) and $\mathrm{N}_{2}$ only (OFF). 
3.8. Ratio of the average current response (IPA to toluene) as a function of the percentage of $\mathrm{OHC} 2 \mathrm{~S}$ ligands on the Au MPCs for A) $4 \%$ and B) $52 \%$ concentration of IPA or Toluene.

4.1. The scheme of exchange reactions for the three thiols, OHC2S, MPA, and MPTMS.

4.2. FTIR spectra of films of C6S coated Au MPCs exchanged with MPA (A) and MPTMS (B) in the vapor phase for various times as indicated.

4.3. Approximation of the exchange kinetics for exchange of $\mathrm{C} 6 \mathrm{~S} \mathrm{Au} \mathrm{MPC}$ with MPA and MPTMS, calculated based on the FTIR peak height of a functional group of the ligands.

4.4. The chemiresistive sensing response to toluene vapor at $52 \%$ saturation, generated by 4 types of sensors we made by a $24 \mathrm{~h}$ vapor phase exchange: C6S Au MPCs, C6S/OHC2S Au MPCs, C6S/MPA Au MPCs, and C6S/MPTMS Au MPCs

4.5. The chemiresistive sensing response to IPA vapor at $52 \%$ saturation, generated by 4 types of sensors we made by a $24 \mathrm{~h}$ vapor phase exchange: C6S Au MPCs, C6S/OHC2S Au MPCs, C6S/MPA Au MPCs, and C6S/MPTMS Au MPCs

4.6. The chemiresistive sensing response to ethanol vapor at $52 \%$ saturation, generated by 4 types of sensors we made by a $24 \mathrm{~h}$ vapor phase exchange: C6S Au MPCs, C6S/OHC2S Au MPCs, C6S/MPA Au MPCs, and C6S/MPTMS Au MPCs

4.7. The chemiresistive sensing response to acetone vapor at $52 \%$ saturation, generated by 4 types of sensors we made by a $24 \mathrm{~h}$ vapor phase exchange: C6S Au MPCs, C6S/OHC2S Au MPCs, C6S/MPA Au MPCs, and C6S/MPTMS Au MPCs

4.8. The response patterns of toluene, IPA, acetone and ethanol vapor provided by the chemiresistive electronic nose consistint of $\mathrm{Au}$ electrode devices coated with films of C6S, OHC2S, MPA, and MPTMS Au MPCs. From left to right: $\mathrm{C} 6 \mathrm{~S}, \mathrm{OHC} 2 \mathrm{~S}, \mathrm{MPA}$ and MPTMS. 


\section{CHAPTER I}

\section{INTRODUCTION}

\subsection{Main Goal/Summary}

The goal of this research was to develop a novel method to vary the functionalization of films of $\mathrm{Au}$ monolayer protected clusters (Au MPCs) via vapor phase place exchange reactions, which is a fast, simple and clean method. Rapid and easy functionalization of Au MPCs is important for several applications, including chemiresistive sensing. This chapter (Chapter I) provides background information on monolayer protected metal clusters, place-exchange reactions, and chemiresistive sensors. Chapter II describes experimental procedures and instrumentation used in this work. Chapter III describes our research on the synthesis of Au MPC films and measurements of the vapor phase place exchange reactions kinetics and effect of functionality on sensor selectivity. Chapter IV describes the use of our method to functionalize multiple sensors for identification of vapor analytes using a sensor array. Chapter V summarizes and provides future directions of this research.

\subsection{Motivaion/Objective}

The motivation behind this research was to develop a simple, fast and versatile method to easily vary the functionalization of Au MPCs by a vapor phase place 
exchange method. Films of Au MPCs have been used for chemiresistive sensing of volatile organic compounds for various applications. It is well-known that the functionality of the MPCs strongly affects the sensor selectivity to different analytes. Our objective was to develop a method to simply and quickly functionalize the MPCs as a film in order to tune the selectivity for a specific analyte or incorporate these sensors into sensor arrays.

\subsection{Synthesis and Functionalization of Au Monolayer Protected Clusters}

$\mathrm{Au}$ nanoparticles have recently gained a great amount of attention due to their fascinating properties and potential applications in a wide range of fields such as vapor sensors, ${ }^{1-4}$ disease diagnostics. ${ }^{5}$ chemical separations, ${ }^{6,7}$ and catalytic applications. ${ }^{8}$ Researchers discovered that $\mathrm{Au}$ nanoparticles (Au NPs) are highly stable and they exhibit unique size-related electronic, magnetic and optical properties. Our research focused on the synthesis of Au MPCs and controlled functionalization. Advances in this area will lead to a better fundamental understanding of structure and property relationships of $\mathrm{Au}$ MPCs and their applications in electronics, sensing and catalysis.

There are generally two methods for synthesizing Au nanoparticles (NPs). The first is by the citrate reduction of $\mathrm{HAuCl}_{4}$ in water at elevated temperature or borohydride reduction of $\mathrm{AuCl}_{4}{ }^{-}$in the presence of citrate. ${ }^{9}$ This method produces $\mathrm{Au}$ nanoparticles electrostatically stabilized by negatively charged citrate ions. The 
second method is known as the Brust-Schiffrin method. ${ }^{10}$ This method is a two-phase synthesis that uses alkanethiolates or other thiol containing molecules as protecting ligands, due to the strong Au-S bond. Aqueous $\mathrm{AuCl}_{4}$ is transferred to toluene and reduced by $\mathrm{NaBH}_{4}$ in the presence of a thiol, forming thiol-coated Au MPCs that are stable in non-polar solutions or when stored in the air as a solid at room temperature.

1.3.1. Citrate reduction method. Turkevich and coworkers ${ }^{9}$ introduced this method in $1951 . \mathrm{HAuCl}_{4}$ was mixed with trisodium citrate in water and heated up to boiling temperature. The metal ion precursor $\left(\mathrm{HAuCl}_{4}\right)$ was reduced to form metal particles which were stabilized by trisodium citrate. The diameter of the particle produced by this method is around $20 \mathrm{~nm}$ (Figure 1.1.).

A later study showed that the particle size could be controlled by varying the metal salt-to-stabilizer ratio between $16 \mathrm{~nm}$ to $147 \mathrm{~nm} .^{11}$ Recently, Yonezawa and coworkers $^{12}$ added sodium 3 -mercaptopropionate as a stabilizer simultaneously with trisodium citrate in the water, which leads to Au NPs of $2.3-12 \mathrm{~nm}$ in diameter, depending on the stabilizer to gold ratio. Electrostatically-stabilized Au NPs could also be used as a seed for growing larger different shaped Au nanostructures, such as nanorods, cubes, disks and branched structures in the presence of cetyltrimethylammonium bromide $(\mathrm{CTAB})^{24,25}$. The Au NPs made by citrate reduction are not stable in solid form. ${ }^{13,14}$ Their tendency to aggregate makes it difficult to produce films by drop-cast deposition. On the other hand, films can be formed with these $\mathrm{Au}$ NPs by cross-linking reactions ${ }^{15}$ with bifunctional groups, charged molecules, or biomolecules to form multilayer films. ${ }^{16}$ 


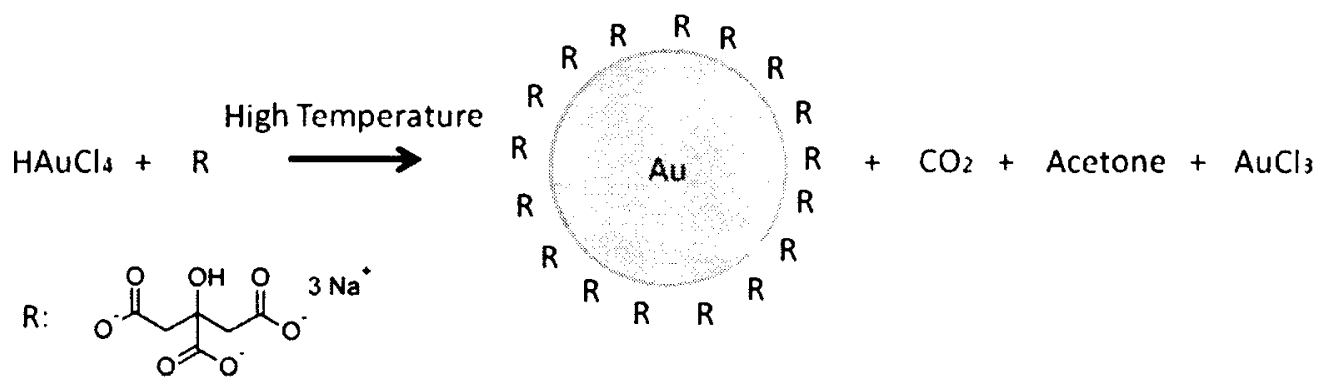

Figure 1.1. Procedure for synthesizing electrostatically-stabilized Au NPs by the citrate reduction reaction. ${ }^{9}$ 
1.3.2. Brust-Schiffrin method. Mulvaney and Giersig ${ }^{17}$ first reported that mercaptan groups (RSH) can be used as a stabilizer for producing Au NPs in 1993. One year later the Brust-Schiffrin method ${ }^{10}$ was published. Briefly, a metal ion precursor $\left(\mathrm{HAuCl}_{4}\right)$ is transferred to a toluene solution by a phase transfer reagent (tetraoctylammonium bromide). In the presence of organomercaptan molecules, which serve as the nanoparticle stabilizer, the ion precursor was reduced by a strong reducing reagent $\left(\mathrm{NaBH}_{4}\right)$, forming $\mathrm{Au}$ NPs with monolayer organic ligands coated on the surface through a strong Au-S bond (Figure 1.2.). The self-assembled monolayer (SAM) coating is much tighter than citrate ions, which can better stabilize the Au NPs and prevent them from aggregation. The nanoparticle size depends on the stabilizer to gold ratio used during the synthesis. The particle diameter ranges from 2 to $5 \mathrm{~nm}$, which is much narrower compared to electrostatically-stabilized Au NPs. This method is widely used to synthesize Au MPCs for the following reasons. First, the clusters are thermally stable in solution and air at room temperature. Also, it is possible to prepare cluster films by drop-cast deposition. Second, the particle size is quite small and has a narrow size dispersity. Smaller particles $(\leq 2 \mathrm{~nm})$ can be produced by quenching the reaction or choosing sterically bulky ligands. A single phase system ${ }^{18}$ was also introduced by Brust for synthesizing p-mercaptophenol protected Au clusters, which opened the field for exploring different functional mercapto group stablilizers, such as mercaptoethanol and mercaptopropionic acid. Murray and coworkers ${ }^{19}$ reported the "place exchange" of thiol ligands by different functional organomercaptans in the liquid phase. Chuanjian et $\mathrm{al}^{20}$ reported coupling reactions on MPCs in order to 


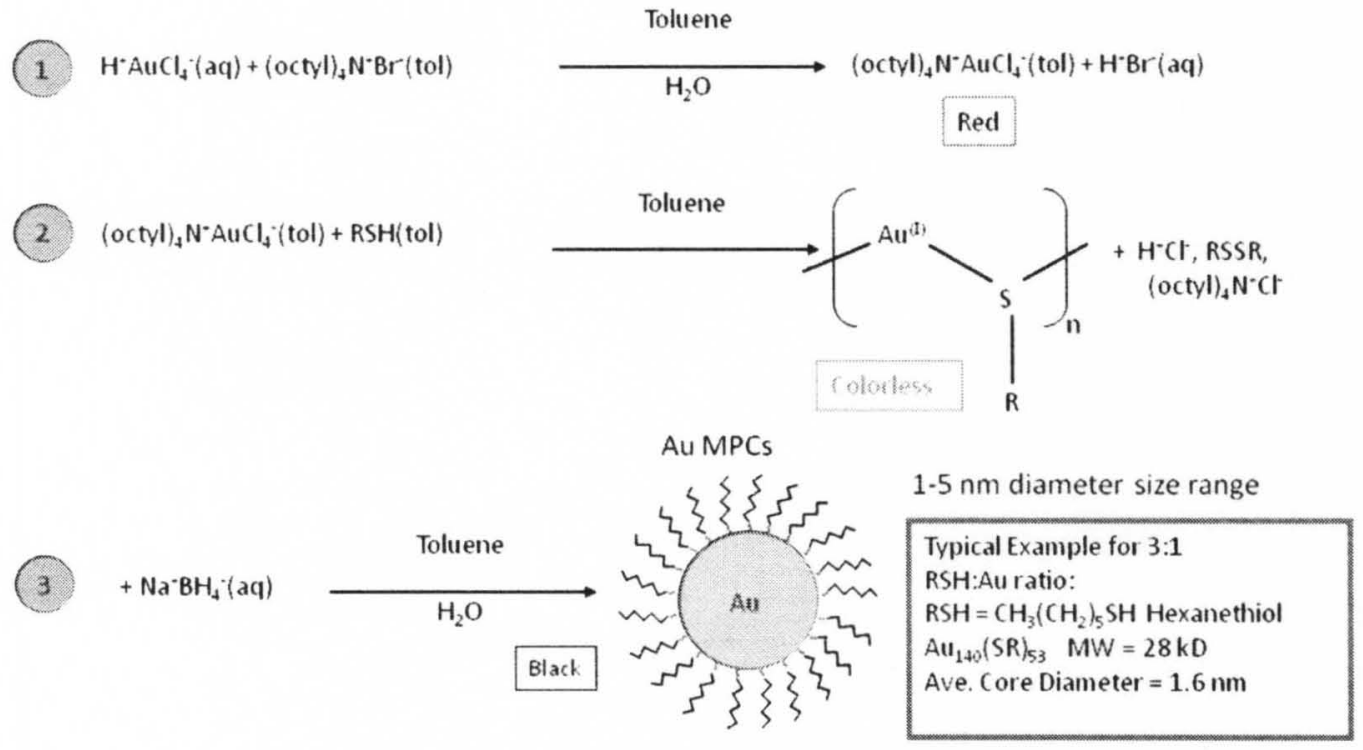

Figure 1.2. Procedure for synthesizing thiol coated Au MPCs through the Brust reaction. ${ }^{10}$ 
further functionalize MPCs.

1.3.3. Place exchange reactions. The place exchange reaction ${ }^{19}$ is a reaction that allows a new thiol (R'SH) to be incorporated onto the MPCs by exchanging with one of the original protecting ligands (RS) on the clusters as follows:

$$
x\left(\mathrm{R}^{\prime} \mathrm{SH}\right)+(\mathrm{RS}) m \mathrm{MPC} \rightarrow x(\mathrm{RSH})+(\mathrm{R} ' \mathrm{~S}) x(\mathrm{RS}) m-x \mathrm{MPC}
$$

where $\mathrm{x}$ is the number of new exchange ligands and $\mathrm{m}$ is the number of original ligands (Figure 1.3.).

This reaction is a useful method to functionalize MPCs and can also be used on CdS nanoparticles protected by arylthiolates ${ }^{21}$ and triphenylphosphine protected $\mathrm{Au}$ MPCs. ${ }^{22}$ The place exchange reactions are usually conducted in the solution phase, but can also occur in the solid or vapor phase. Murray and co-workers ${ }^{19}$ studied the mechanism and dynamics of place exchange reactions on Au MPCs in the solution phase. They discovered that the exchange reaction pathway begins with the penetration of the new ligand into the protecting monolayer followed by desorption of the original protecting ligand and then attachment of the incoming ligand to the vacancy left by the original one. The exchange reaction usually takes place at active defects, such as a vertex or edge sites of the cluster. The terrace regions, on the other hand, are almost inert to the solution phase exchange reaction. In the solution phase place exchange reaction, the original ligands become protonated as a free thiol in solution. Disulfides or other oxidized sulfur species are not involved in the reaction. The rate and extent of the reaction can be controlled by the R'SH to RS mole ratio, the solubility, and the molecular size of R'SH versus RSH. Qun and coworkers ${ }^{23}$ reported 


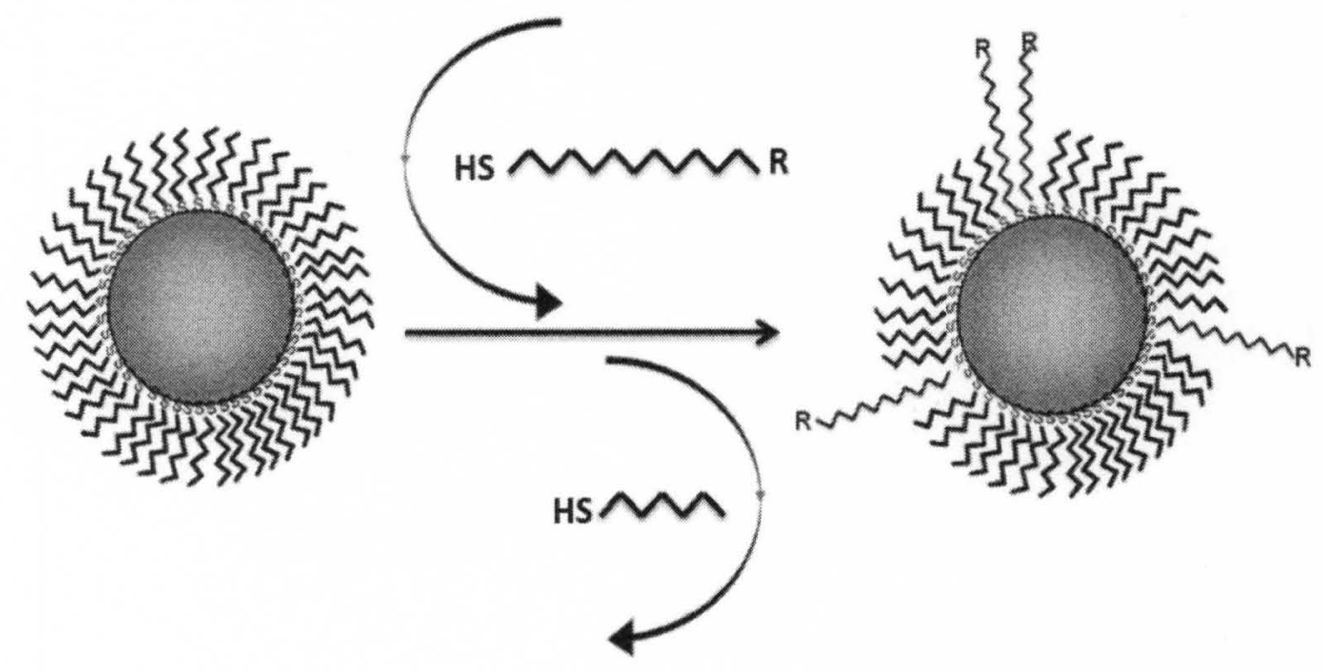

Figure 1.3. General scheme for the place exchange reaction between Au MPCs and various functionalized thiols, where $\mathrm{R}=\mathrm{Br}, \mathrm{CN}, \mathrm{Ph}, \mathrm{OH}, \mathrm{COOH}, \mathrm{F}, \mathrm{Si}\left(\mathrm{OCH}_{3}\right)_{3}$, etc. ${ }^{19}$ 
a place exchange reaction in the solid phase. They used a polymer solid support functionalized with a thiol molecule to hold the Au MPCs. The thiol molecule exchanged with the attached ligand molecule on the Au MPCs, they rinsed it off from the polymer, and produced newly functionalized Au MPCs. Through solid phase place exchange, they could exchange on to a specific site without affecting other protecting ligands. Crooks and coworkers ${ }^{26}$ demonstrated that the self-assembly of alkanethiol monolayers on a $\mathrm{Au}$ surface could be achieved both through liquid or vapor phase deposition as follows:

$$
\mathrm{Au}+\mathrm{RSH} \rightarrow \mathrm{Au}\left(\delta^{+}\right) / \mathrm{HS}\left(\delta^{-}\right) \mathrm{R}
$$

In related work, Lewis and coworkers ${ }^{27}$ detected organomercaptan vapors using thin films of alkylamine coated Au MPCs. They discovered that the alkylamine coated $\mathrm{Au}$ MPCs showed an irreversible electronic response toward thiol vapors, indicating that the original alkylamine ligand were replaced by thiols in the vapor phase. These discoveries indicate that place-exchange reactions for Au MPCs could also be performed in the vapor phase. Here we will use a vapor phase exchange method to change the functionalization of Au MPCs.

\subsection{Electronic Properties of Au MPC Films}

Films of Au MPCs can be used as chemiresistors. These films conduct by an electron hopping mechanism ${ }^{28,29,30}$ according to the following equation:

$$
\sigma_{E L}\left(\delta_{e^{\prime}} T\right)=\sigma_{0} \exp \left[-\beta_{d} \delta_{e}\right] \exp \left[-E_{a} / R T\right]
$$

Where $\sigma_{\mathrm{EL}}$ is the conductivity of the MPC film, $\sigma_{0}$ is a pre-exponential tunneling 
factor, $\delta_{\mathrm{e}}$ is the distance between particles, $\beta_{\mathrm{d}}$ is a quantum mechanical tunneling factor, $R$ is the ideal gas constant, $T$ is the temperature, and $E_{\mathrm{a}}$ is the activation energy barrier which uncharged particles need to overcome in order to be positively or negatively charged during electron transport. Figure 1.4. illustrates electron conduction through the MPC film. In the Au MPC film, the metal Au cores are separated by the organic monolayer insulator, but the electron can still jump or hop over the organic layer to the metal centers because the insulator is very thin $(1-2 \mathrm{~nm})$. As shown in the equation, the conductivity $\left(\sigma_{\mathrm{EL}}\right)$ is exponentially dependent on the metal particle edge-to-edge distance $\left(\delta_{\mathrm{e}}\right)$. The longer chain length of the coating ligands, the longer the distance between metal cores and lower conductivity. The conductivity also depends on the tunneling factor $\beta_{\mathrm{d}}$, which is related to the electronic interaction between the positive and negative site (donor and acceptor). For example, n-alkanethiols have a large $\beta_{d}$ compared to $\omega$-hydroxyalkanethiol. ${ }^{63} E_{a}$ is the activation energy, which describes the energy needed to transfer an electron between two neutral nanoparticles. It depends on the dielectric of the coating, particle size, and particle spacing as follows: ${ }^{31,32}$

$$
\mathrm{E}_{\mathrm{A}} \approx \mathrm{N}_{\mathrm{A}} \mathrm{e}^{2} \delta_{\text {edge }} / \varepsilon_{\mathrm{S}} \varepsilon_{0} \mathrm{R}_{\text {core }}\left(\mathrm{R}_{\text {core }}+\delta_{\text {edge }}\right)
$$

where $\mathrm{N}_{\mathrm{A}}$ is Avogadro's number, $\mathrm{e}$ is the electronic charge, $\delta_{\text {edge }}$ and $\mathrm{R}_{\text {core }}$ are the distance between the nanoparticle edges and radius of the particle, respectively, $\varepsilon_{S}$ is the static dielectric constant of the coating, and $\varepsilon_{0}$ is the permittivity of free space. $\varepsilon_{S}$ describes the dielectric property of the surrounding. A larger $\varepsilon_{S}$ value results in a 


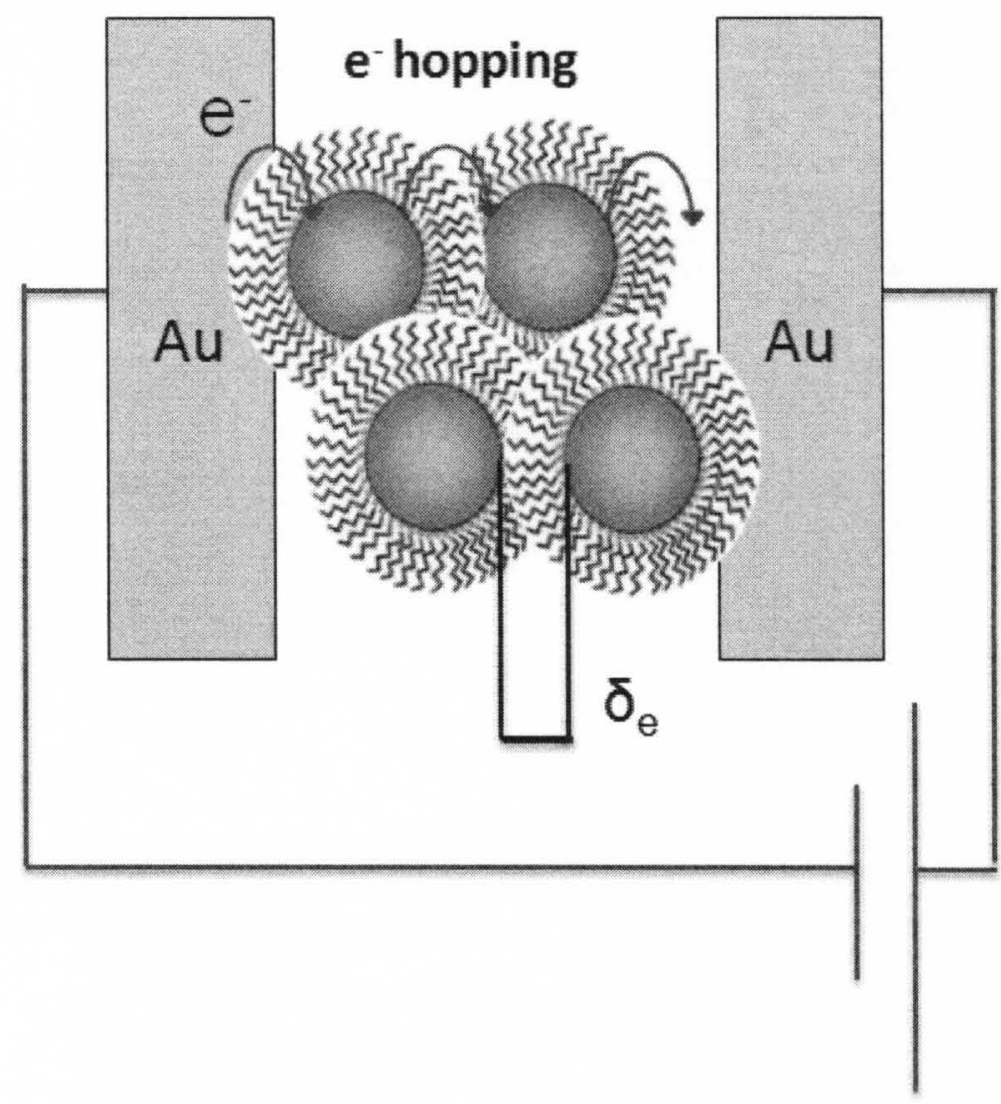

Figure 1.4. Scheme for electron hopping mechanism. Electron hops over between the metal cores when potential is applied. $\delta_{\mathrm{e}}$ is the separating distance between metal cores. $^{28}$ 
decrease in $\mathrm{E}_{\mathrm{A}}$ and a conductivity increase.

For a continuous metal, the conductivity decreases with increasing temperature. For a film of Au MPCs, the conductivity increases with increasing temperature. ${ }^{102,103}$ An evaluation of the temperature dependent conductivity (Arrhenius plot) allows an evaluation of $\mathrm{Ea} .^{102,103}$

\subsection{Chemiresistive Sensing}

Chemiresistors are materials whose conductivity changes in the presence of a chemical analyte of interest. This type of sensor consists of a chemiresistor between two electrodes. In the sensing experiment, a current or resistance is measured while applying a constant voltage between the electrodes. The current remains stable until exposure to a chemical analyte. The interaction between the chemiresistor and the analyte causes a resistance change, which further leads to a current change (Figure 1.5.). There are several materials that can be used as chemiresistors, such as metals, ${ }^{33}$ metal oxides ${ }^{33-36}$, polymers ${ }^{37}$ and metal embedded into polymers. ${ }^{38}$ Different materials exhibit different chemiresistive mechanisms. For example, Pd metal detects hydrogen gas by $\mathrm{Pd}$ hydride $(\mathrm{PdHx})$ formation in the presence of hydrogen. ${ }^{39}$ The pristine $\mathrm{Pd}$ 


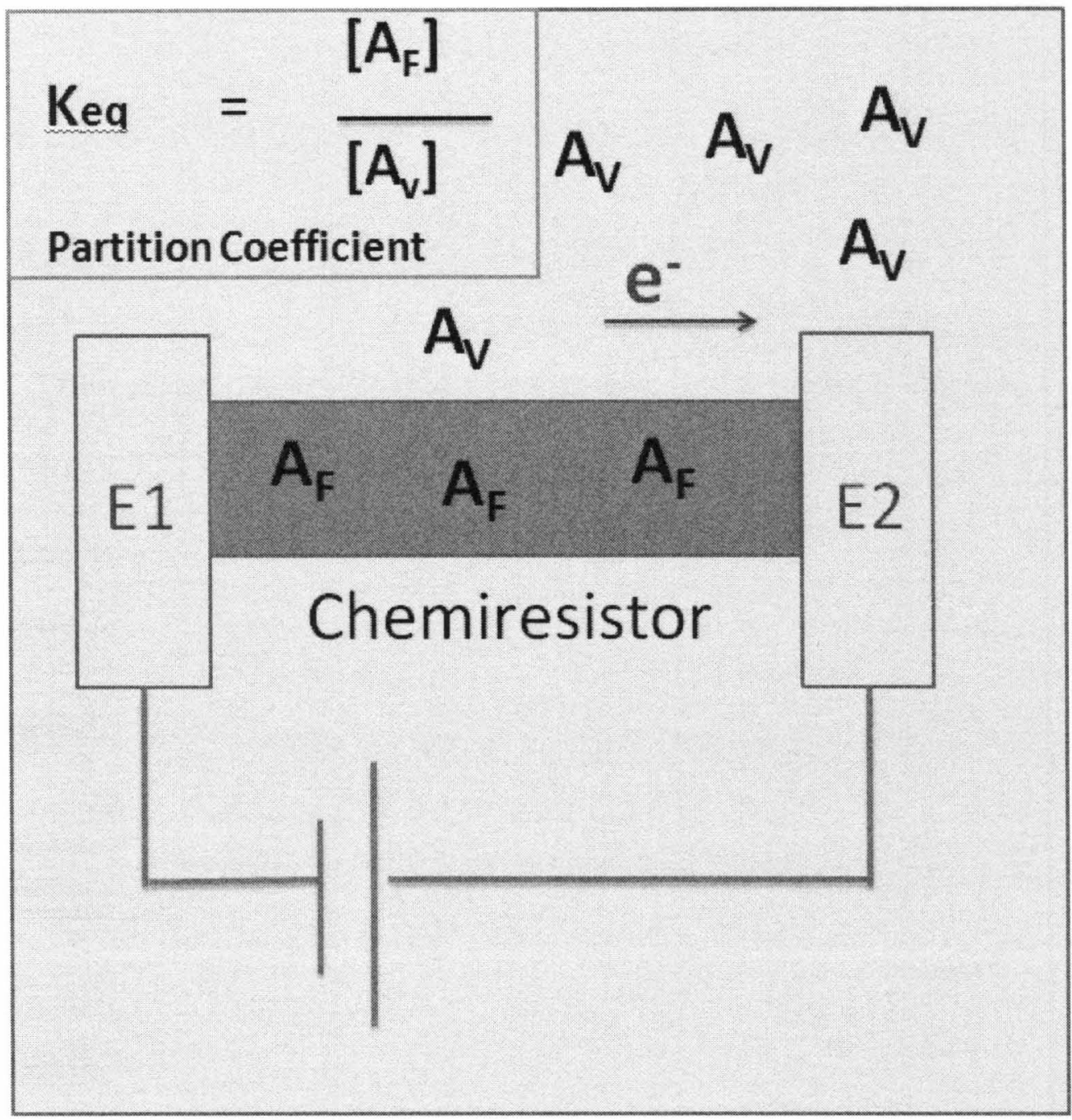

Figure 1.5. Scheme of a chemiresistive sensor. $A_{V}$ : analytes in environment (vapor phase). $A_{F}$ : analytes in chemiresistor (film). When the device is exposed to the analytes, the interaction between the chemiresistive transducer and the analyte changes the conductivity of the chemiresistor and generates a current signal. ${ }^{1}$ 
has a smaller resistance compared to $\mathrm{PdHx}$. When exposed to hydrogen gas, the $\mathrm{H}_{2}$ molecule adsorbs and penetrates into the $\mathrm{Pd}$ metal, forming $\mathrm{Pd}$ hydride, which increases the resistance. ${ }^{40}$ Chemiresistors comprised of p-type or n-type semiconductors ${ }^{33,36}$ will either change the electron or hole density when exposed to a specific chemical analyte and change the resistance of the semiconductor.

The detection of volatile organic compounds (VOCs) is important for numerous applications, including public safety, ${ }^{41,42}$ diagnostics, ${ }^{5}$ environmental protection, ${ }^{43,44}$ and homeland security. ${ }^{45}$ Wohltjen and Snow ${ }^{1}$ first reported the use of Au MPCs as chemiresistors for sensing VOCs. They drop-cast deposited films of octanethiolate coated $\mathrm{Au}$ MPCs onto electrodes for VOC detection, such as 2-propanol, tetrachloroethylene, and toluene. Their result showed that in the presence of the vapor analyte, the current decreased drastically for toluene, less for 2-propanol, and no change for water. They also suggested that the selectivity towards different vapor analytes could be varied by changing the functional groups on the protecting ligands.

Later, Evans and coworkers ${ }^{46,47}$ synthesized $\mathrm{Au}$ MPCs with $\mathrm{OH}, \mathrm{COOH}, \mathrm{NH}_{2}$, and $\mathrm{CH}_{3}$ groups for detection of polar and nonpolar VOCs. They discovered that films of MPCs that have ligands with $\mathrm{CH}_{3}$ as the ending group respond to nonpolar vapors such as pentane and hexane better than the others, but do not respond well to polar vapors, such as methanol and ethanol. 


\subsection{Electronic Noses}

Electronic noses have gained tremendous attention in the sensor field during the past two decades because of their wide spread applications in diverse fields. Dodd and Persaud $^{48}$ introduced the electronic nose concept in 1982, which is a device that consists of several different sensors with different responses to vapor phase analytes. They used three different metal oxide vapor sensors and detected several analytes by measuring the steady-state current of these sensors. Pattern recognition methods ${ }^{49,50,51}$ can be used to distinguish the vapor phase analytes. The goal of the electronic nose is to mimic the olfaction of living species with an instrument and generate several descriptors for certain analytes. ${ }^{64}$ One of the key advantages over traditional sensors is that the electronic nose can detect the analytes in a mixture. ${ }^{52-55}$ Since the idea of the electronic nose was introduced, much work has been done in order to understand and organize the olfactory system and descriptor receptor cell. ${ }^{64}$ On each receptor cell, only one odorant descriptor can be generated and located. ${ }^{64}$ Different molecules have different functional groups which lead to different odorant signal generation by each receptor cell. ${ }^{64}$ Several receptors activated differently result in a unique descriptor pattern that determines the odor impression of the molecule ${ }^{64}$ Electron nose devices have such components provided by Gardner (Figure 1.6): ${ }^{56}$

1. An aroma delivery system which removes the volatile analytes from the sample to the sensor array system.

2. Sample chamber where the sensor arrays are located. This place is where the sensor detects the vapor analytes by chemical or physical interactions. 


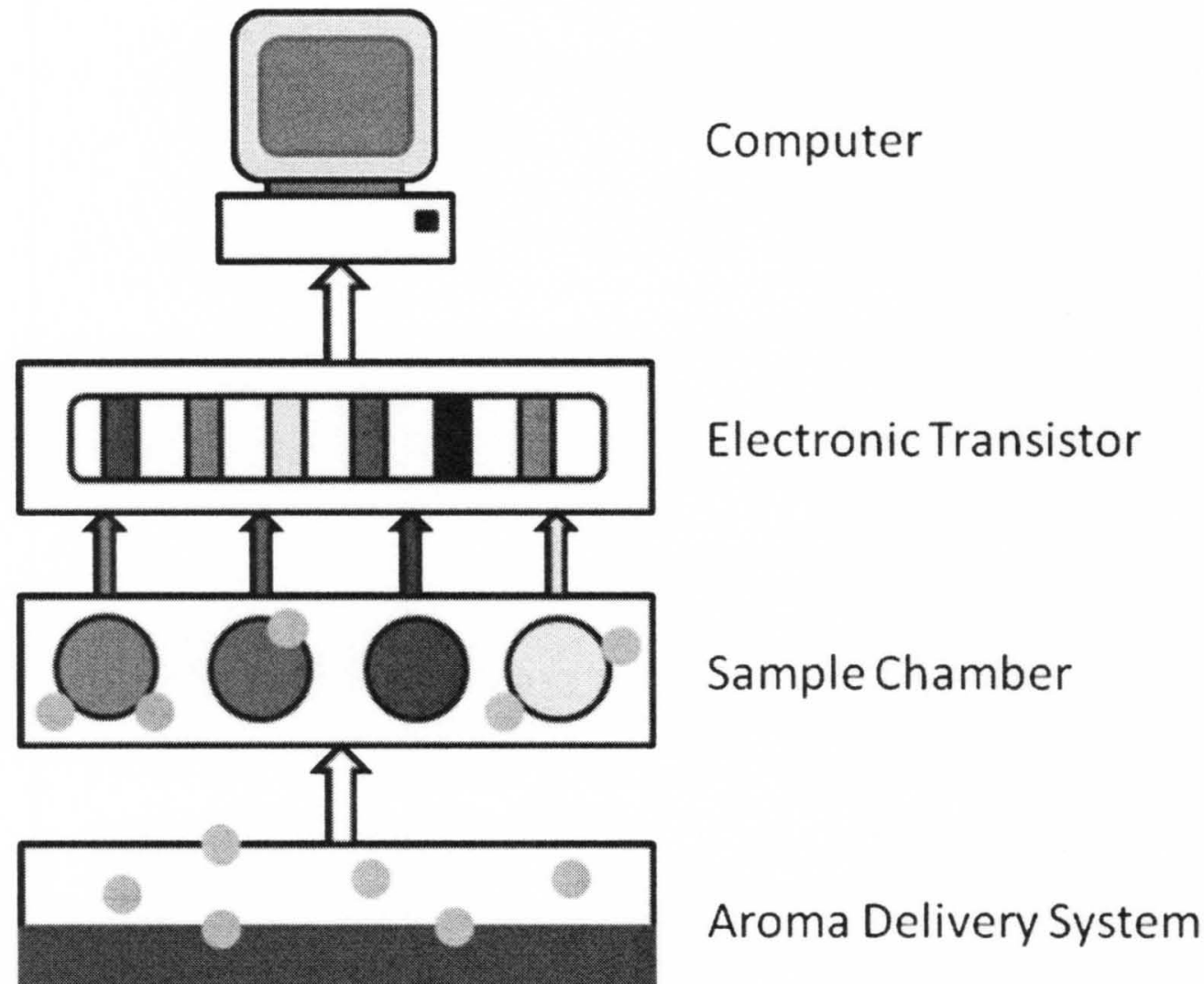

Figure 1.6. Scheme for basic electronic nose instrument setup. The electronic nose systems simulate the human olfactory system, resulting in the recognition of volatile molecules by pattern recognition analysis. ${ }^{56}$ 
3. Electronic transducer which converts the chemical information into an electronic signal.

4. Computer to digitalize the electronic signal and analyze the data. This is the last step of the process where the identification and classification of the analytes occurs.

The sensor array transducer is a key part of the electronic nose device. A good sensor should fulfill several requirements. First, the sensitivity towards target analyte or group should be high, It is best to have a detection limit similar to the human nose $\left(10^{-12} \mathrm{~g} / \mathrm{mL}\right) .{ }^{57}$ Second, the sensor should have the capability to detect and differentiate a wide range of chemicals. In other words, it should have good selectivity. Sometimes, we need the electronic nose to quantify the detectable chemicals. The capability to quantitatively detect the target is important. In addition, a fast response and recovery time is needed in order to have high sensing efficiency. Up to now there are many sensors that can be utilized as a transducer, including metal oxide sensors, ${ }^{58,59}$ conducting polymer sensors, ${ }^{60}$ and piezoelectric acoustic wave sensors. ${ }^{61}$ In our research, we use a film of Au MPCs as a chemiresistive transducer for potentially building an electronic nose. As we discussed before, the film of Au MPCs is a good chemiresistor which can detect vapors by monitoring an electrical resistance change. The analyte partitions into the $\mathrm{Au}$ MPC film and alters the resistance through a change in the distance $\delta_{\mathrm{e}}$ or $E_{\mathrm{a}}$ through a change in the dielectric of the environment. Clearly, it depends on the interaction between the protecting layer of the Au particles and the target analyte. Different analytes and different protecting ligands have different interactions, which will generate different signals. Clearly, the film of Au MPCs with 
different functionalizations can be used as a receptor cell to generate certain descriptors.

\subsection{Summary}

In this chapter we reviewed the synthesis and functionalization of $\mathrm{Au} N P s, \mathrm{Au}$ MPCs, their electronic properties, and the chemiresistive sensing applications. $\mathrm{Au}$ NPs or Au MPC synthesis can be achieved mainly by 2 methods, in the presence of citrate or by the Brust method. ${ }^{10} \mathrm{Au}$ NPs stabilized by monolayer organomercaptan groups synthesized by the two-phase Brust reaction generally have smaller size and size dispersity. Also, Au MPCs synthesized this way are more stable than those made by citrate reduction. People can make cluster films by the drop-cast method or inking without $\mathrm{Au}-\mathrm{Au}$ aggregation. In order to further functionalize Au MPCs, researchers introduced place-exchange reactions to replace the original protecting ligands. Au MPCs can be used for many applications, including sensors, energy, separations and catalysis. Our research focuses on chemiresistive sensing applications. Up to now, the most common application for chemiresistive sensing with films of Au MPCs is for detecting volatile organic compounds (VOCs). The sensing properties depend on several factors, such as particle size, type of monolayer, ligand spacing, film thickness, and flexibility. ${ }^{65}$ Electron conduction through the Au MPC films occurs by an electron hopping mechanism. ${ }^{28}$ The conductivity depends on the distance between the edges of the Au core and the dielectric properties of the environment. When the film is exposed to the target vapor analyte, the analyte can partition into the film and change the 
conductivity by altering the distance between Au particles and dielectric properties of the environment. It has been shown that the polarity determines the extent of the analyte partitioning. ' Polar monolayers coating the Au MPCs have a better affinity for polar vapors. Films of $\mathrm{Au}$ MPCs stabilized by polar organic ligands are more selective to polar vapors and vice versa. The sensitivity of the cluster films depends on the flexibility and film thickness. Generally, Au MPCs coated with a longer chain length monolayer are more flexible and contains more volume for analyte adsorption. Thinner films usually have better sensitivity due to a larger active surface to volume ratio. ${ }^{66}$ The metal core size also plays an important role in the sensing activity. ${ }^{67,68}$ Though the cluster film can distinguish the polarity of the analytes with same concentration, the selectivity is far from good enough to identify and quantify the analytes, especially when the target analytes are mixed with other vapor compounds. In order to further enhance the selectivity, electronic noses which consist of Au MPC film sensor arrays with different functionalizations have been fabricated to detect the type and amount of analyte. ${ }^{47}$ By combining each of the sensing responses generated by the sensor cells, one can get the specific response pattern for the analyte. Pattern recognition was used to identify the analyte. ${ }^{50}$ More importantly, the electronic noses can identify and quantify the analyte in a mixure. ${ }^{68}$ Au MPCs can be coupled with GC to enhance the separation. ${ }^{6,7}$ 


\section{CHAPTER II}

\section{EXPERIMENTAL}

In this chapter we describe the experimental procedures and instruments used, including: 1) Synthesis of C6S Au MPCs, 2) Drop-cast deposition, 3) Vapor phase place exchange, including a) salt plates for FTIR and NMR studies, b) interdigitated array (IDA) of electrodes for conductivity and sensing studies, c) vary film thickness, and d) varying thiol molecules, and 4) Characterization by a) FTIR, b) NMR, c) conductivity, and d) chemiresisitive sensing.

\subsection{Synthesis of Hexanethiol-Coated Au Monolayer Protected Clusters(MPCs)}

We synthesized hexanethiolate-coated gold monolayer-protected clusters (C6S $\mathrm{Au}$ MPCs) by the Brust method. First, $0.957 \mathrm{~g}$ of $\mathrm{HAuCl}_{4} \cdot 3 \mathrm{H}_{2} \mathrm{O}$ was dissolved in 25 $\mathrm{mL}$ of water and $2.18 \mathrm{~g}$ of $\mathrm{TOABr}$ was dissolved in $100 \mathrm{~mL}$ of toluene. The two solutions were mixed and stirred for 20 minutes until all of the $\mathrm{AuCl}_{4}{ }^{-}$transferred into the toluene phase. The toluene phase was separated and $1.12 \mathrm{~mL}$ of hexanethiol, corresponding to a 3:1 thiol:Au ratio, was added to the toluene and stirred until the solution became colorless. The solution was cooled in an ice bath to $\sim 0^{\circ} \mathrm{C}$ and a 10 -fold excess of $\mathrm{NaBH}_{4}(1.01 \mathrm{~g}$ in $10 \mathrm{~mL}$ of water) was added to the toluene solution 
with fast stirring. The solution turned black within a few seconds, indicating the formation of metallic Au MPCs. An additional $10 \mathrm{~mL}$ of water was added to the solution and stirred overnight. The toluene layer was separated and removed by rotary evaporation. The remaining black sludge Au MPC product was suspended in $200 \mathrm{~mL}$ of acetonitrile and collected on a glass fritted Büchner funnel by filtration. After this, we washed the black solid product with an additional $250 \mathrm{~mL}$ of acetonitrile and 100 $\mathrm{mL}$ of ethanol and thoroughly dried before collecting. Au MPCs prepared are $1.6 \pm$ $0.4 \mathrm{~nm}$ in diameter according to literature. ${ }^{70}$

\subsection{Au IDA Electrode Device Fabrication and Drop Cast Deposition}

The IDA electrode device was fabricated in a clean-room facility at the University of Louisville by photolithography, sputtering and lift-off procedures (Figure 2.1.). Two Au microelectrodes were sputtered or evaporated over a $\mathrm{Si} / \mathrm{SiOx}$ substrate and patterned with a $23 \mu \mathrm{m}$ separation. Contact wires were attached to the Au contact pads with $\mathrm{Ag}$ epoxy and cured at $80^{\circ} \mathrm{C}$ overnight. Next, a coating of Torr-seal epoxy was placed over the Ag epoxy under the same condition. The electrodes were cleaned by rinsing with acetone and IPA and dried under $\mathrm{N}_{2}$ gas. After rinsing and drying, the electrodes were placed in a UVO ozone cleaner (Jelight Company Inc., Irvine, CA) for 15 min before Au MPC film deposition.

Hexanethiolate-coated (C6S) Au MPCs were dissolved in toluene with a concentration $\sim 30 \mathrm{mg} / \mathrm{mL}$ and sonicated for 2 minutes. A $1 \mathrm{~mL}$ solution was drop-cast deposited onto a $\mathrm{KBr}$ salt plate and air dried to make the film for FTIR analysis. This 
A

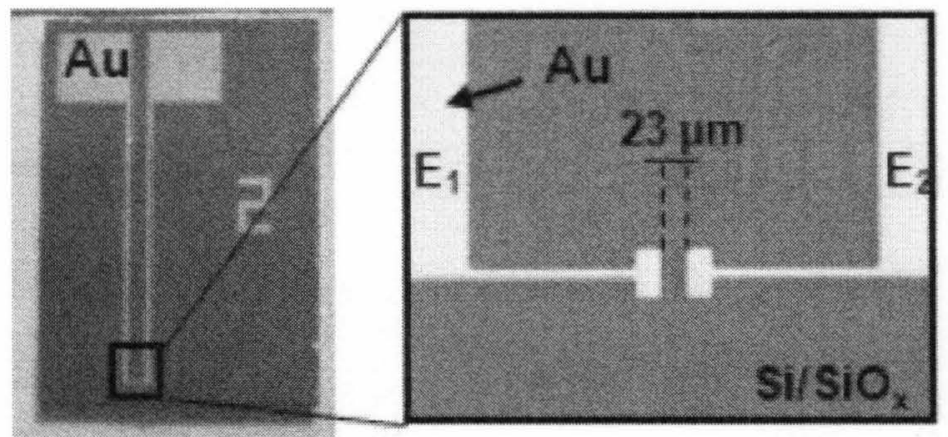

B

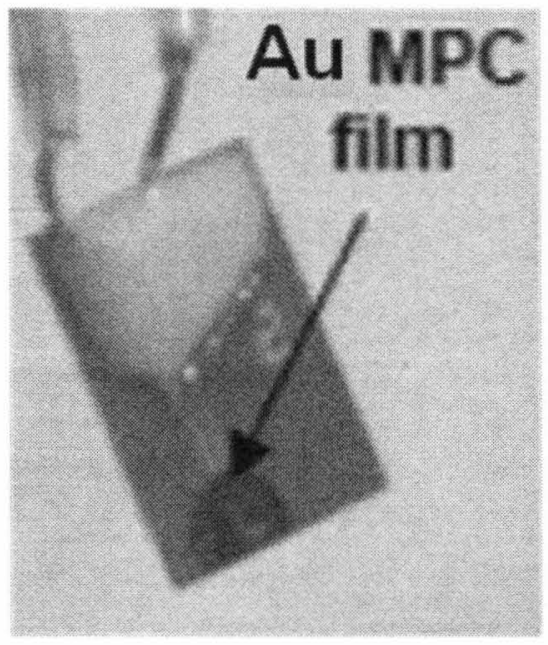

Figure 2.1. A: Blow up of $23 \mu \mathrm{m}$ gap region of the IDA electrode. B: C6S Au MPC film drop cast deposited on the IDA electrode.(adapted from reference ${ }^{62}$ ) 
film was later removed from the salt plate for NMR analysis. 2 drops of the solution were used to deposit a thin film on the IDA electrodes for conductivity and chemiresistive sensing measurements, as shown in figure 2.1.B.

\subsection{Vapor Phase Place Exchange}

Figure 2.2 shows our set up for the vapor phase place exchange reactions. In a petri-dish, we placed the thiol of interest for exchange in the middle in a plastic vial. A film of $\mathrm{C} 6 \mathrm{~S} \mathrm{Au}$ MPCs were drop-cast deposited onto a $\mathrm{KBr}$ salt plate and IDA electrode placed in the petri-dish for studying the exchange kinetics and chemiresistive sensing, respectively as described in the following sections.

In order to exchange the C6S ligands on the Au MPCs with a different ligand, the exchange ligand should be volatile. In our experimental design, we choose mercaptoethanol (OHC2S), 3-mercaptopropionic acid (MPA), and mercaptopropyltrimethoxy-silane (MPTMS) as the exchanging ligands to replace the C6S ligands on the cluster. In the case of mercaptoethanol, we put $\sim 1 \mathrm{~mL}$ in a small plastic holder, and it was put in a closed petri-dish to concentrate the vapor molecule which will replace the $\mathrm{C} 6 \mathrm{~S}$ ligands through vapor phase place exchange.

\subsection{Chemiresistive Vapor Sensing Set Up}

Figure 2.3 shows the chemiresistive vapor sensing set up we used. First, we 


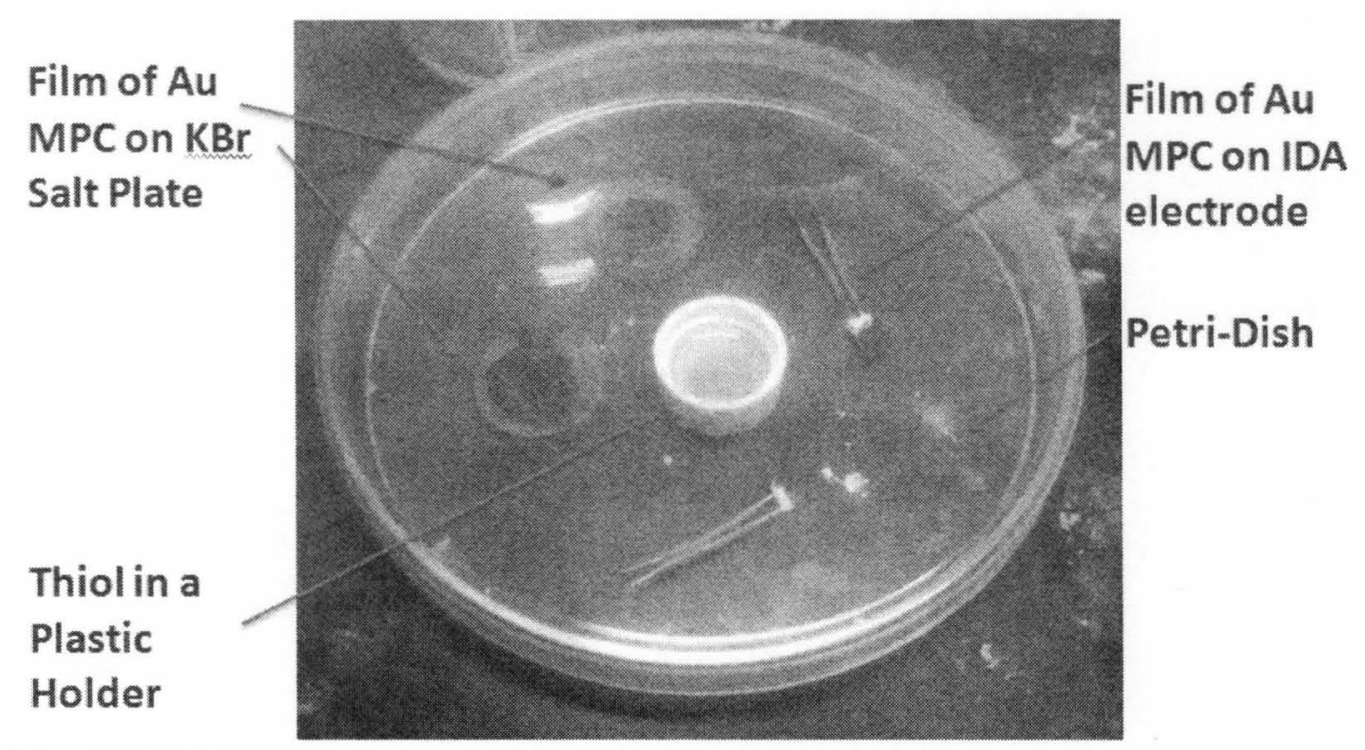

\begin{tabular}{|l|l|}
\hline Thiols & Structures \\
\hline Hexanethiol & Mercaptoethanol \\
\hline Mercaptopropyltrimethoxy-silane & \\
\hline Mercaptopropionic acid & \\
\hline
\end{tabular}

Figure 2.2. Scheme of the experimental set up for the vapor phase place exchange reactions (top): we put thiol exchange reagent, film of Au MPC on salt plate and electrode in a covered petri-dish. Table (botton): thiols we used as the exchange reagents. 
separate the nitrogen gas into two lines with a T-junction and controlled the flow rate by two different flow meters. One line was pure $\mathrm{N}_{2}$ and the other bubbled through the solvent of the vapor of interest, which was isopropanol (IPA), toluene, ethanol (EtOH), or acetone. The two lines were recombined into one line with a T-junction and the mixed $\mathrm{N}_{2}$ and vapor $/ \mathrm{N}_{2}$ flowed over the sample. On/Off valves were used to expose the sample to pure $\mathrm{N}_{2}$ or vapor $/ \mathrm{N}_{2}$ at various concentrations.

The chemiresistive vapor sensing experiment was performed with a $\mathrm{CH}$ Instruments 660A (Austin, TX) electrochemical workstation operating in chronoamperometry (CA) mode, which monitors the current as a function of time. One Au electrode of the device was connected to the working electrode lead of the potentiostat and the other was connected to both the counter and reference electrode leads together. The current was monitored at a potential of $0.3 \mathrm{~V}$ while the device was exposed to alternating flows of $\mathrm{N}_{2}$ or $\mathrm{N}_{2}$ /vapor mixture. The different vapor concentrations were achieved by mixing the vapor $\mathrm{N}_{2}$ and pure $\mathrm{N}_{2}$ at different flow rates controlled by Cole Parmer flow meters and operated by a three-way valve. The sensing response was collected by monitoring the current as a function of time. Before and after exposure as shown in Figure 2.4. The response of the sensor was calculated as:

$$
\text { Response }=\left(i-i_{b}\right) / i_{b}
$$




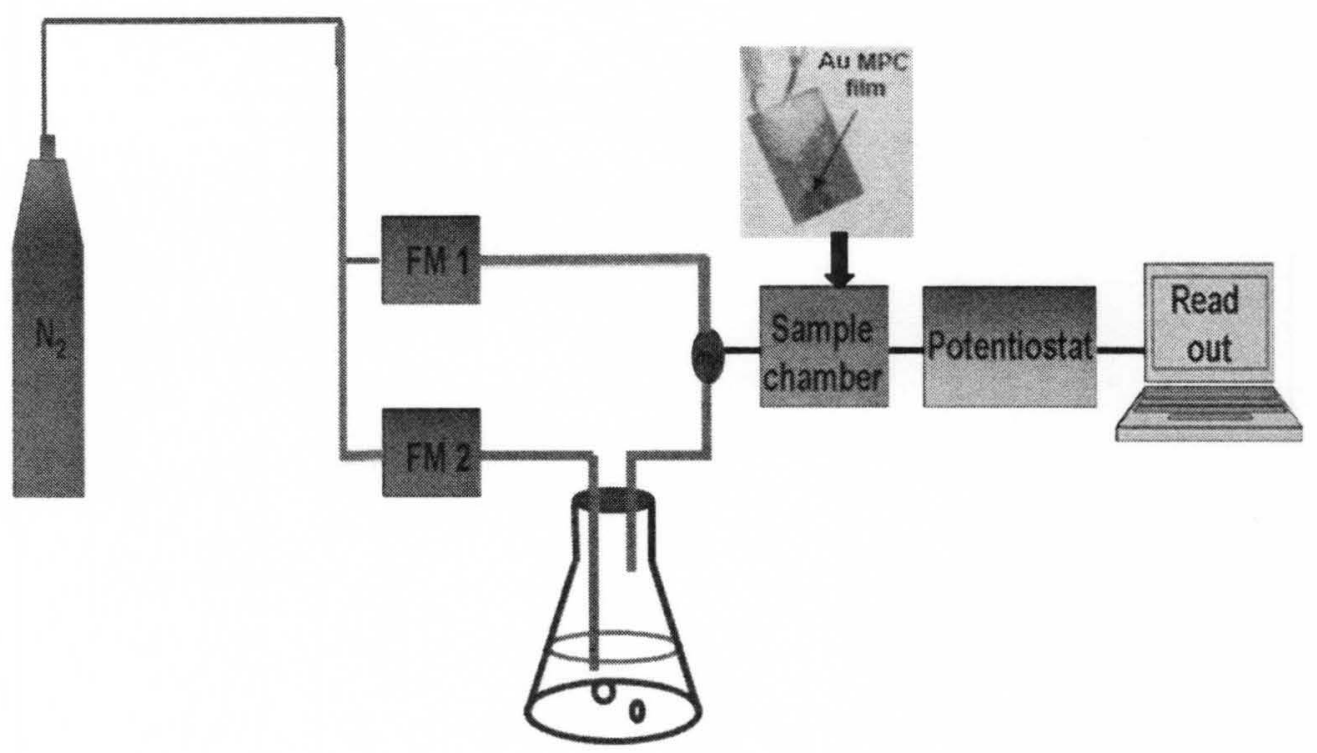

Figure 2.3. The chemiresistive vapor sensing set up with the film of Au MPCs in the sample chamber. $\mathrm{N}_{2}$ gas has been separated into two lines (pure $\mathrm{N}_{2}$ and $\mathrm{N}_{2} /$ vapor) and recombined before flows into the sample chamber. 


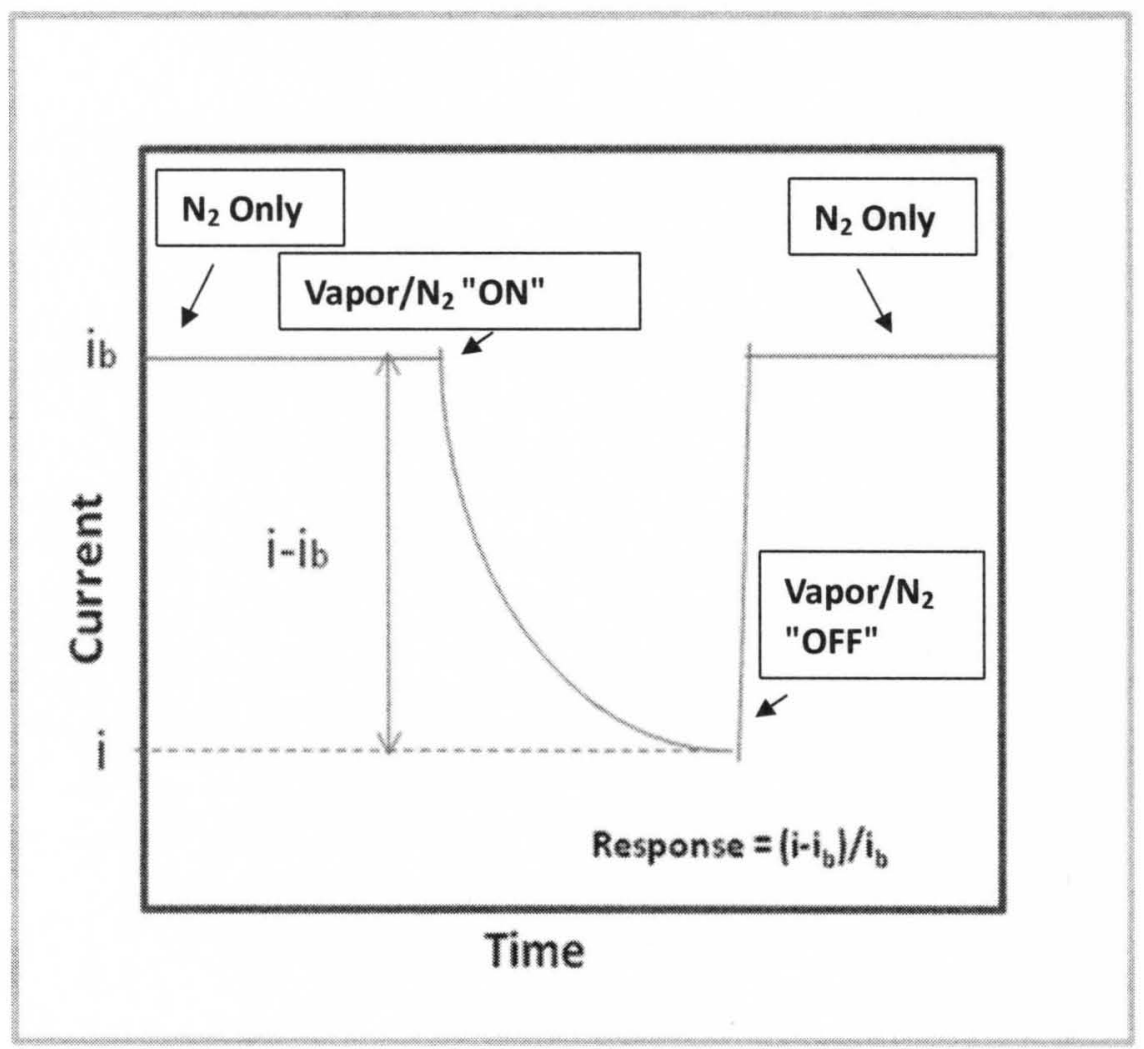

Figure 2.4. Typical chemiresistive sensing plot and response calculation. 


\subsection{Spectroscopy Measurement}

2.5.1. FTIR measurements. $\mathrm{KBr}$ salt plate was chosen as substrate for the measurement and FTIR instrument (FTS 7000 SERIES, DIGILAB) was use for measuring spectra. After background measurements of the pristine $\mathrm{KBr}$, we drop-cast deposited a film of C6S Au MPCs onto the salt plate using $20 \mathrm{mg} / \mathrm{mL}$ toluene MPC solution and air dried. After characterization we placed the salt plate with Au MPC film into the vapor exchange device for several times and removed, then we blew $\mathrm{N}_{2}$ gas on it to remove the left over vapors for 30 min and measure the FTIR spectrum at room temperature. We measured the spectrum after different exchange times and the exchange kinetics was studied based on the peak height ratio of each corresponding functional group of the ligands at various times of exchange. For example, in the experiment of exchanging $\mathrm{C} 6 \mathrm{~S}$ ligands with $\mathrm{OHC} 2 \mathrm{~S}$, we measured the peak height corresponding to the $\mathrm{CH}_{3}$ and $\mathrm{OH}$ asymmetric stretch and the ratio of the ligands $\mathrm{C} 6 \mathrm{~S}$ to $\mathrm{OHC} 2 \mathrm{~S}$ was estimated by the ratio of the $\mathrm{CH}_{3}$ to $\mathrm{OH}$ peak height.

2.5.2. NMR measurements. After FTIR measurement, the film of Au MPCs was rinsed off with chloroform-d solution and a small amount of iodine was added to the solution and sonicated for 30 minutes, causing the ligands to be removed from $\mathrm{Au}$ and dissolved into the disulfides as follows:

$$
\mathrm{Au}_{\mathrm{x}}(\mathrm{SR})_{\mathrm{y}} \stackrel{\text { Iodine }}{\rightarrow} \mathrm{xAu}+\mathrm{y} / 2(\mathrm{RSSR})
$$

The chloroform-d solution was removed from the solid $\mathrm{Au}$ for measuring NMR spectrum and the solid Au was discarded. The ligand ratio was determined by the corresponding groups. The steps are shown in Figure 2.5. 

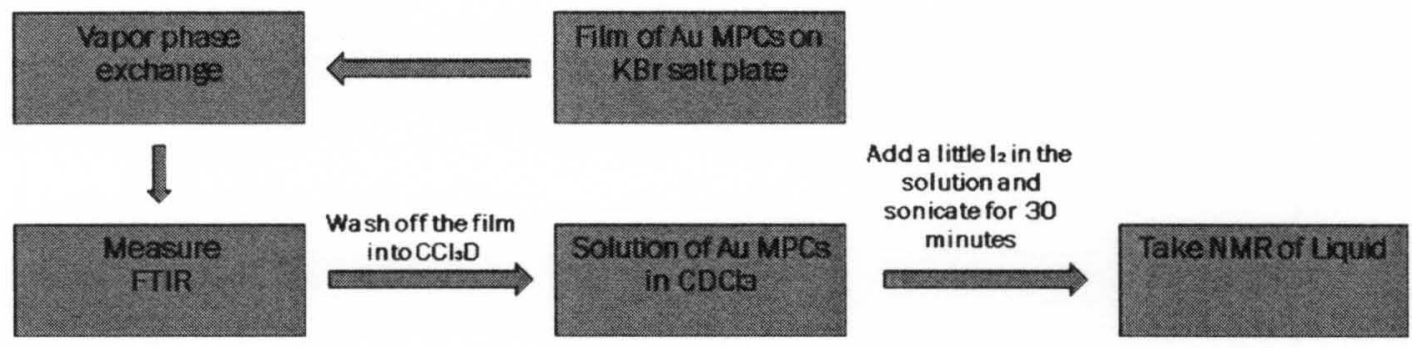

Figure 2.5. Scheme of the FTIR and NMR measurements. A film of Au MPCs was drop-cast deposited on to a $\mathrm{KBr}$ salt plate and placed into the exchange device for several times and removed for the FTIR and NMR measurements. 


\section{CHAPTER III}

\section{VAPOR PHASE THIOL-PLACE EXCHANGE REACTIONS ON FILMS OF GOLD MONOLAYER-PROTECTED CLUSTERS FOR SELECTIVE SENSING OF VOLATILE ORGANIC COMPOUNDS}

Here we describe a new method to quickly vary the functionalization of films of Au monolayer protected clusters (Au MPCs) via vapor phase thiol-place exchange reactions. In the procedure, we first synthesize hexanethiolate-coated gold monolayer-protected clusters (C6S Au MPCs) and then prepare a film by drop-cast deposition. Placing the film of C6S Au MPCs into a closed container with mercaptoethanol leads to the replacement of the hexanethiolate ligands with mercaptoethanol ligands by vapor phase place-exchange. FT-IR and NMR spectroscopy data show the exchange kinetics. After one day of exchange, up to 91 percent of the hexanethiolates $(\mathrm{C} 6 \mathrm{~S})$ were replaced with mercaptoethanol (OHC2S). The resistance of the films decreased from $250 \mathrm{M} \Omega$ to $150 \mathrm{M} \Omega$ after the one-day exchange. Importantly, the chemiresistive sensing response ratio of isopropanol (IPA) versus toluene (TOL) changed from 0.3 (IPA/TOL) for C6S Au MPCs to 1.8 after exchange with mercaptoethanol (C6S/OHC2S Au). This shows that our method is useful for easily and rapidly altering the functionality of the films in order to change 
the selectivity of these materials as chemiresistive sensors for volatile organic compounds (VOCs).

\subsection{Introduction}

For many years, researchers have been interested in the detection of volatile organic compounds(VOCs) ${ }^{71-73,101}$ because it has many important applications in numerous areas, such as food industry, ${ }^{74,75}$ environmental protection, ${ }^{43,44}$ bomb detection, ${ }^{45}$ and disease diagnostics. ${ }^{5}$ In the food industry, for example, the detection of VOCs given off by the food could be operated by a human nose. But in other fields, many VOCs are toxic to humans. In the last decade, people have developed many sensing techniques to identify and quantify VOCs, including optical sensors, ${ }^{76,77}$ electrochemical sensors, ${ }^{78}$ acoustic wave devices, ${ }^{61}$ piezoelectric devices,${ }^{80}$ GC-MS, ${ }^{81}$ and others. A miniaturized sensor for the detection of VOCs is termed "electronic nose", ${ }^{48-55}$ which is a simple device that combines the output signal of selective, cross reactive chemical sensors with data analysis software. It is simpler than GC-MS and much cheaper. Chemiresistive materials can be applied as transducers to detect VOCs. A chemiresistor is a simple, sensitive and rugged material used to detect chemicals in the gas or liquid phase. The detection is achieved by monitoring the electrical resistance change in the material caused by interactions with the analyte of interest. In our lab, we use thin films of Au monolayer protected clusters (Au MPCs) as chemiresistive transducers to detect VOCs. 
Au MPCs consist of two parts, a metallic $\mathrm{Au}$ core that is surrounded by a monolayer of organic ligands which is usually alkanethiolates. Wohltjen and Snow first reported that metal MPCs could be used for sensing VOCs. ${ }^{1}$ They found that the resistance of drop-cast films of $\mathrm{Au}$ MPCs changed in the presence of various VOCs, including 1-propanol, toluene, and water. Later, reports showed that different functionalized MPCs such as aromatic thiols, carboxylate thiols, and alcohol thiols have different sensing selectivity towards various VOCs. ${ }^{46,47}$ Electron conduction through metal MPCs occurs by an electron hopping mechanism, which depends on the distance between clusters and dielectric of the environment. In the presence of VOCs, the target vapor molecule will be adsorbed into the film and change one of these variables, leading to a resistance change. The magnitude of the resistance change and the sensing mechanism depends on the characteristics of the protecting ligands and analyte. Generally, films with non-polar ligand have better affinity towards non-polar analytes and vice-versa. ${ }^{46,47}$

In order to vary the functionality of MPCs, researchers usually synthesize a batch of $\mathrm{Au}$ MPCs with variable functionalities or they thiol place-exchange reaction. In the place-exchange reaction, a new thiolate ligand replaces the original ligands surrounding the MPCs. The place exchange reaction was conducted in MPC solutions. In the typical procedure, the MPC solution and new ligand are stirred for several days and the MPCs purified by removing solvent, filtering and drying prior to characterization. Here we describe the use of vapor phase place-exchange reactions to quickly and easily functionalize the Au MPC films to alter the chemiresistive sensing 
selectivity. Specifically, we describe the vapor phase place exchange of hexanethiol-coated (C6S) Au MPCs with mercaptoethanol (C2SOH) ligands. We monitored the exchange process with NMR and FT-IR spectroscopy over a 48 hour period and tested the chemiresistive sensing selectivity towards IPA and TOL before and after the exchange at various times. The benefit of vapor phase exchange is that only one batch of Au MPCs needs to be synthesized, but many functionalities can be made quickly and easily. It is versatile and functionalities can be used that are not easy or possible in solution.

\subsection{Experimental}

3.2.1. Chemicals: Hexanethiol (HT, 96\%), mercaptoethanol (ME 99\%), sodium borohydride $(99 \%)$, tetraoctylamonium bromide ( $\mathrm{TOABr}, 98 \%$ ), toluene ( $\mathrm{Tl} 99.9 \%$ ), and isopropanol (IPA 99.9\%) were purchased from commercial sources and used as received. Hydrogen tetrachloroaurate $\left(\mathrm{HAuCl}_{4} \cdot 3 \mathrm{H}_{2} \mathrm{O}\right)$ was synthesized from pure $\mathrm{Au}$. Barnstead Nanopure water $(\mathrm{R} \geq 17.8 \mathrm{M} \Omega-\mathrm{cm})$ was employed for all aqueous solutions.

3.2.2. Synthesis: Hexanethiolate-coated gold monolayer-protected clusters (C6S Au MPCs) were synthesized according to the Brust reaction. as described in Chapter II.

3.2.3. Vapor exchange setup: The vapor exchange setup was described in Chapter II.

3.2.4. Vapor sensing experiment: Refer to Chapter II. 


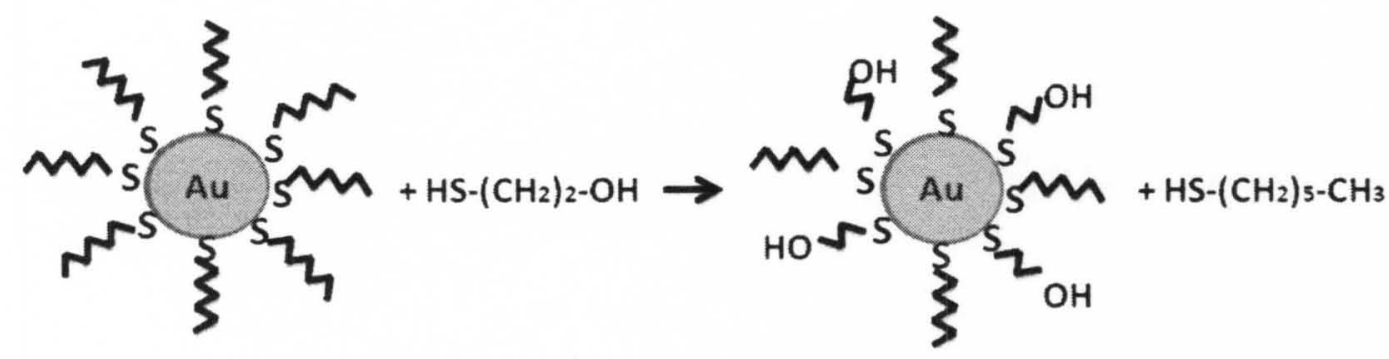

Figure 3.1. Scheme of vapor phase exchange on film of C6S Au MPCs using OHC2S as exchange reagent. In the vapor phase, the $\mathrm{OHC} 2 \mathrm{~S}$ replaced the original $\mathrm{C} 6 \mathrm{~S}$ ligand leading to $\mathrm{C} 6 \mathrm{~S} / \mathrm{OHC} 2 \mathrm{~S} \mathrm{Au} \mathrm{MPCs}$ formation. 


\subsection{Results and Discussion}

3.3.1.Vapor Phase Place-Exchange Reaction Kinetics. Figure 3.1 illustrates the set up for the vapor phase place exchange reaction. In a petri-dish we place the new ligands for exchange (OHC2S) in the middle in a plastic vial cap. $\mathrm{C} 6 \mathrm{~S}$ coated $\mathrm{Au}$ MPCs films were drop cast deposited on a $\mathrm{KBr}$ salt plate and IDA electrode and they were placed in the petri-dish for the exchange kinetics and sensing study, respectively.

Figure 3.2. shows the results of FTIR analysis of films of MPCs during various stages of the vapor phase place exchange. Figure 3.2. shows a series of FTIR spectra of a film of C6S Au MPCs after $0,1,4,24$ and 48 hours of exchange with OHC2S. The peak around $3300 \mathrm{~cm}^{-1}$ corresponds to the $\mathrm{OH}$ stretch of the incoming $\mathrm{OHC} 2 \mathrm{~S}$ ligand and the peak at $2956 \mathrm{~cm}^{-1}$ corresponds to the asymmetric $\mathrm{CH}_{3}$ stretch of the $\mathrm{C} 6 \mathrm{~S}$ ligand being replaced. It is a shoulder peak of the asymmetric $\mathrm{CH}_{2}$ stretch at $2920 \mathrm{~cm}^{-1}$. For quantification, we very roughly esimated the height of the $\mathrm{CH}_{3}$ peak, neglecting the height from the asymmetric $\mathrm{CH}_{2}$ stretch and compared that to the height of the $\mathrm{OH}$ stretch. The FTIR spectra show that the $\mathrm{OH}$ stretch increased in intensity with exchange time while the $\mathrm{CH}_{3}$ stretch intensity decreased, as expected for exchange of C6S ligands with $\mathrm{OHC} 2 \mathrm{~S}$.

Figure 3.3 shows the corresponding NMR results, obtained as described in section 2.5. Chapter II. The peak at $0.81 \mathrm{ppm}$ corresponds to the $\mathrm{CH}_{3}$ protons of the C6S ligands and the peak at $3.67 \mathrm{ppm}$ corresponds to the $\mathrm{CH}_{2}$ next to the sulfur of $\mathrm{C} 6 \mathrm{~S}$. These two peaks decreased with increasing exchange time as expected for loss of $\mathrm{C} 6 \mathrm{~S}$ 


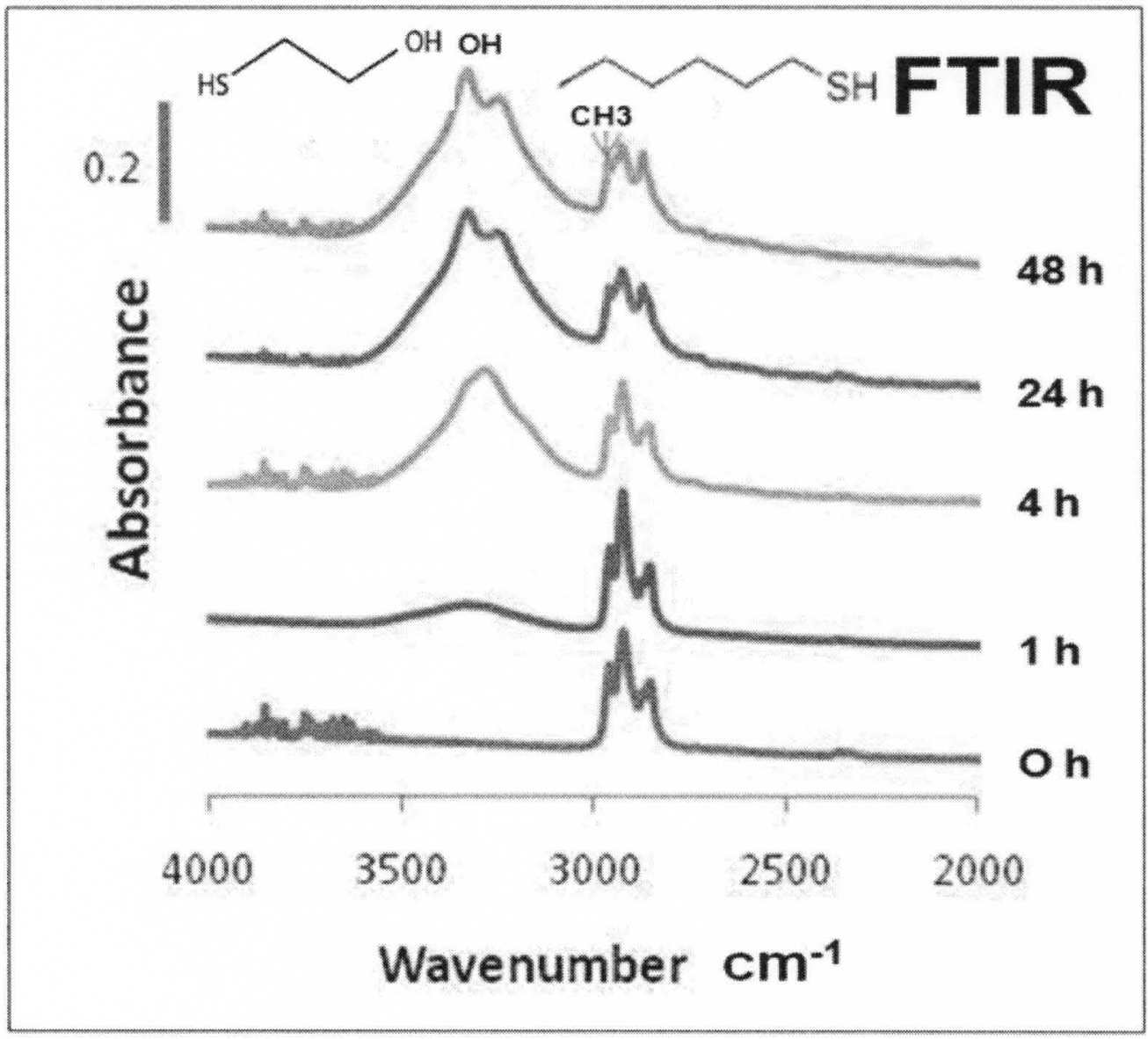

Figure 3.2. A series of FTIR spectra of OHC2S exchange for $0,1,4,24$, and $48 \mathrm{~h}$.

FTIR analysis indicated that C6S ligands were gradually replaced by $\mathrm{OHC} 2 \mathrm{~S}$ ligands. 


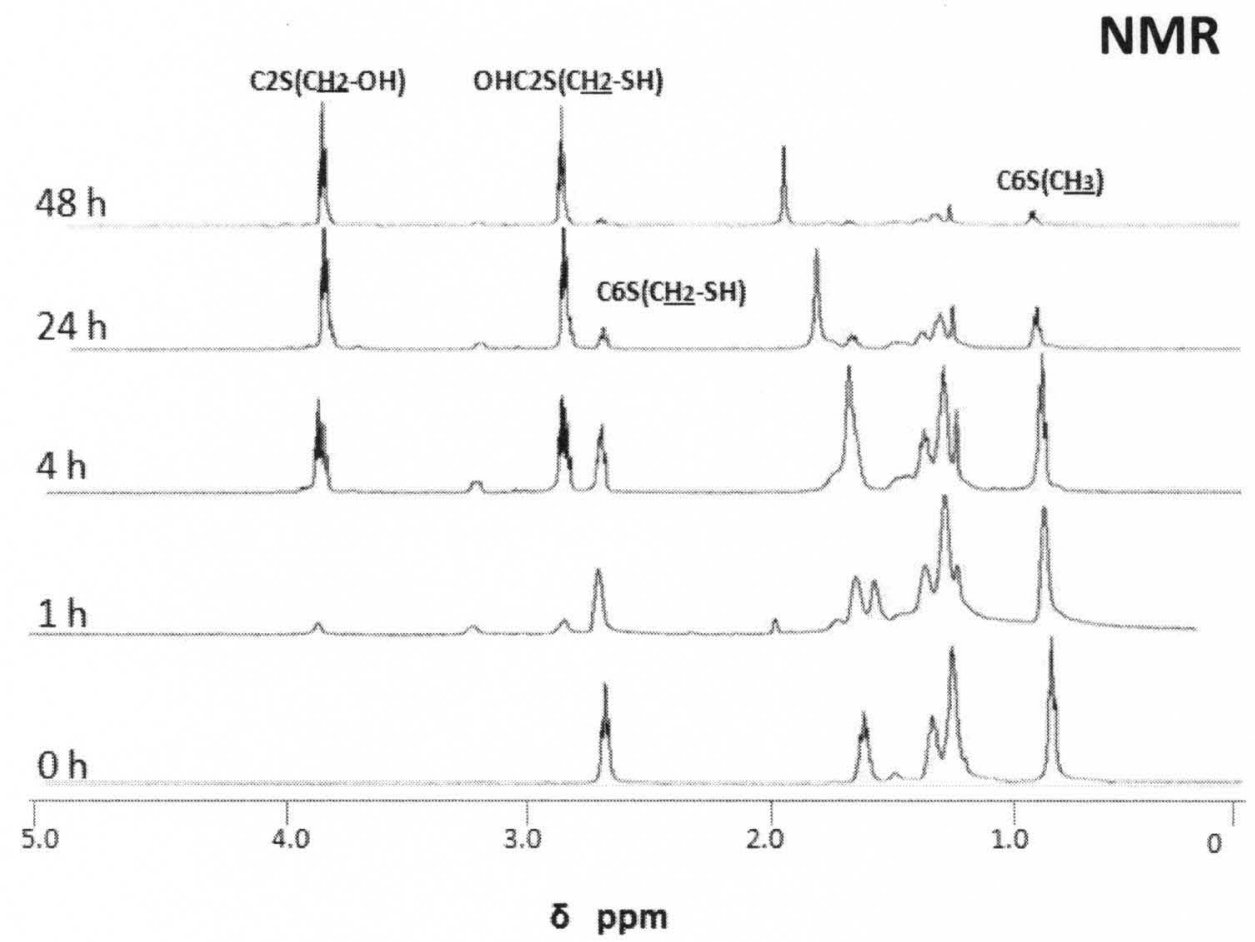

Figure 3.3. A series of NMR spectra of $\mathrm{OHC} 2 \mathrm{~S}$ exchange for $0,1,4,24$, and $48 \mathrm{~h}$.

NMR analysis indicated that $\mathrm{C} 6 \mathrm{~S}$ ligands were gradually replaced by $\mathrm{OHC} 2 \mathrm{~S}$ ligands. 
ligands. The peak at $2.85 \mathrm{ppm}$ corresponds to the $\mathrm{CH}_{2}$ adjacent to the $\mathrm{S}$ of $\mathrm{OHC} 2 \mathrm{~S}$ and the peak at $3.85 \mathrm{ppm}$ corresponds to the $\mathrm{CH}_{2}$ adjacent to the $\mathrm{OH}$ of $\mathrm{OHC} 2 \mathrm{~S}$. These two peaks increased with increasing exchange time. The NMR shows that the OHC2S ligands replace $\mathrm{C} 6 \mathrm{~S}$ ligands in the vapor phase, which is consistent with the FTIR, but more quantitative. We calculated the percentage of $\mathrm{OHC} 2 \mathrm{~S}$ ligands on the Au MPCs based on the FTIR peak height and integrated NMR peaks, as a function of exchange time, as shown in Figure 3.4. The two methods showed similar results. Because FTIR is faster and simpler compared to NMR, this shoes that we can use FTIR peak heights to estimate the ligand ratio.

We monitored the exchange for 3 samples by FTIR. Two were performed on the same day and one on a different day. The two on the same day were reproducible but significantly different from the third sample on a different day, shown in Figure 3.5. We attribute this to a different film thickness on the two days. Experiments later showed that the exchange rate decrease as the MPC film thickness increases as shown in Figure 3.6. We made the film in 4 aluminum caps with same bottom area. The film thickness variation was controlled by changing the number of drops deposited in those caps from one drop to four drops. They were air dried and then we placed them all in the petri-dish for vapor phase exchange for 4 hours. The solution used for drop cast deposition was $\sim 50 \mathrm{mg} / \mathrm{mL}$. 


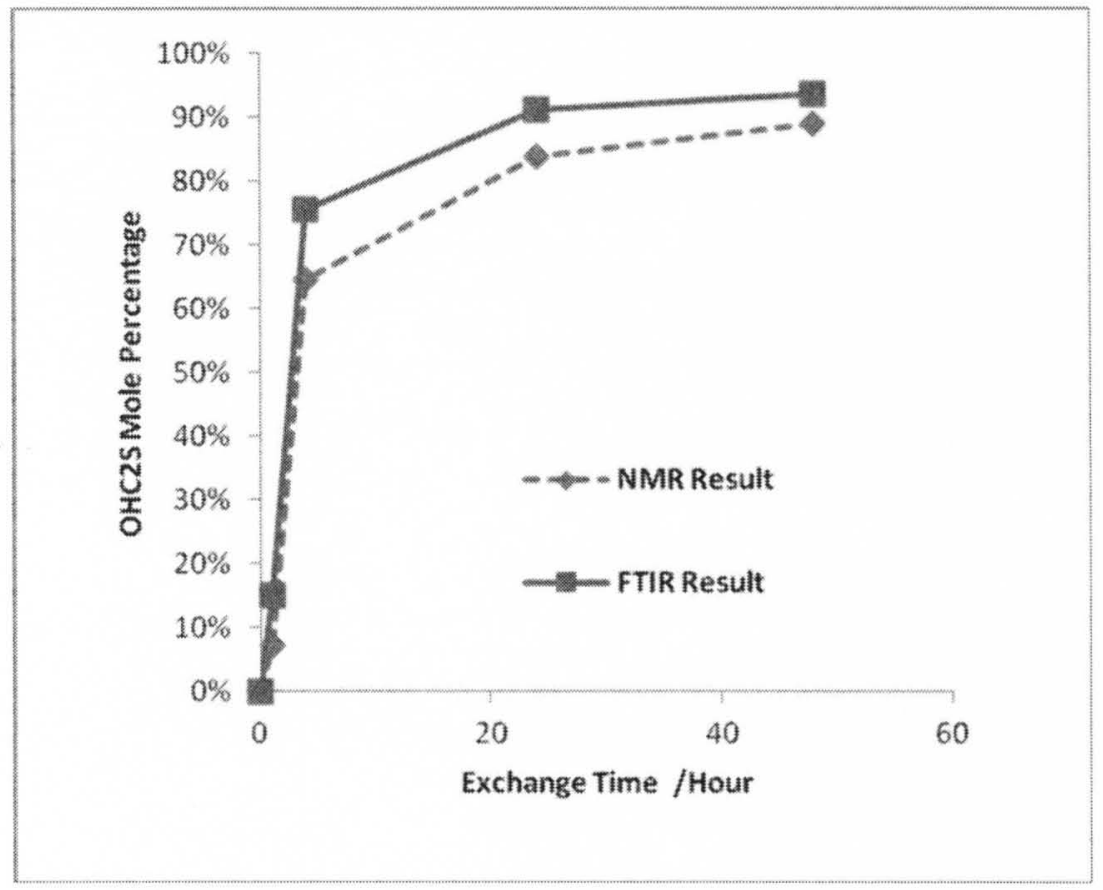

Figure 3.4. NMR and FTIR comparison for the OHC2S exchange ratio during the vapor phase place exchange. 


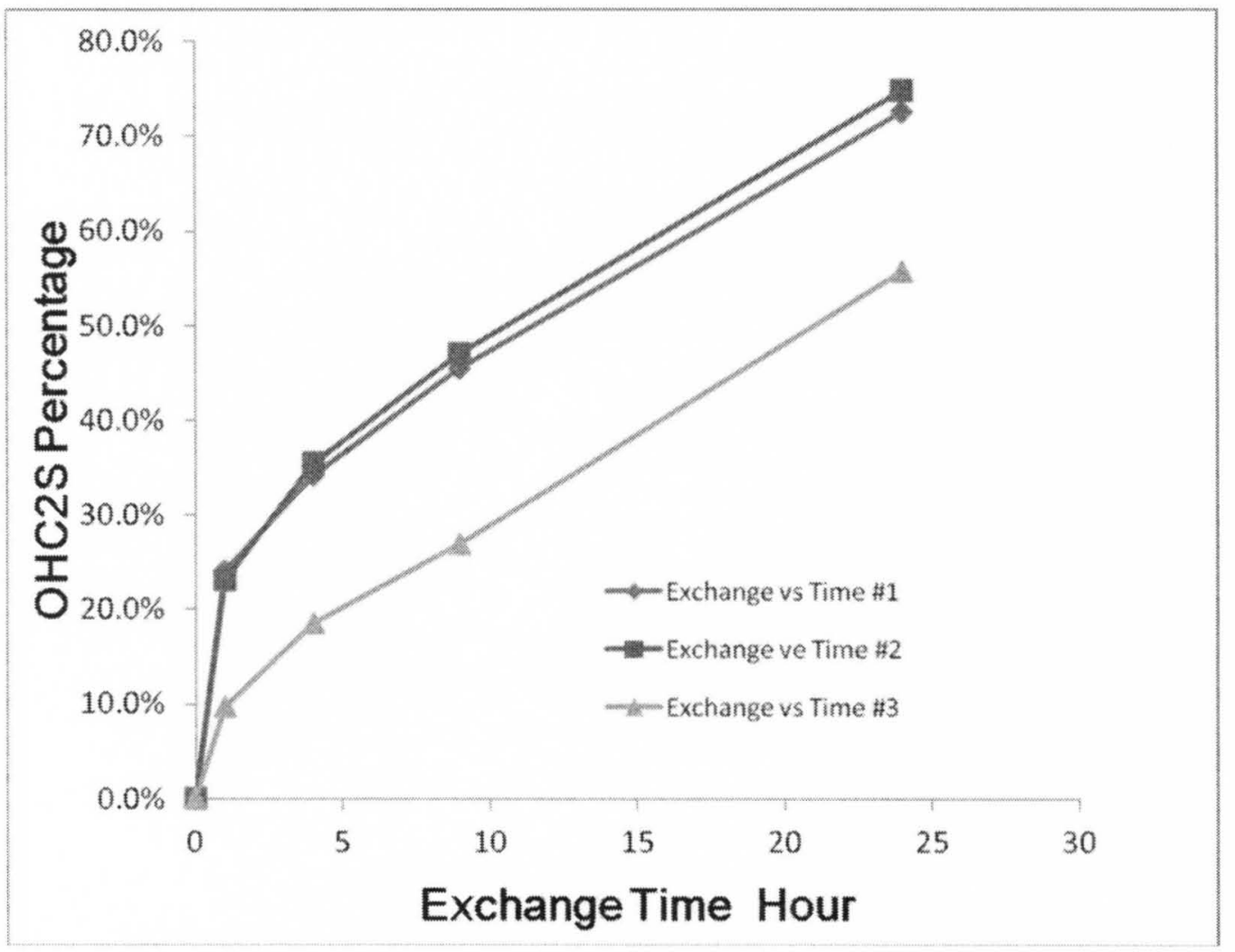

Figure 3.5. 3 FTIR samples on 2 different day. Sample \#1 and \#2 were performed on the same day and sample \#3 performed on a different day. 


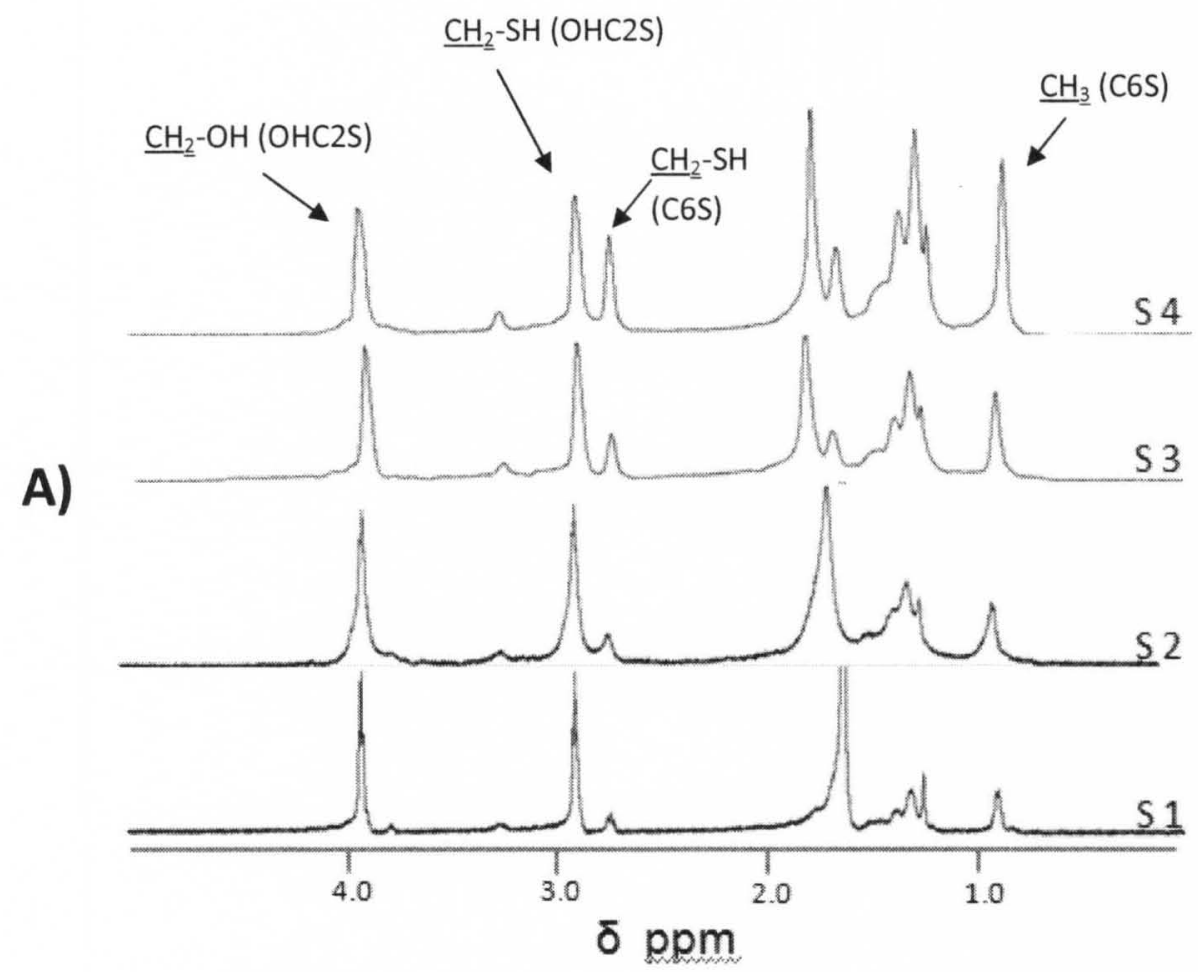

S4

\section{2 \\ 1}

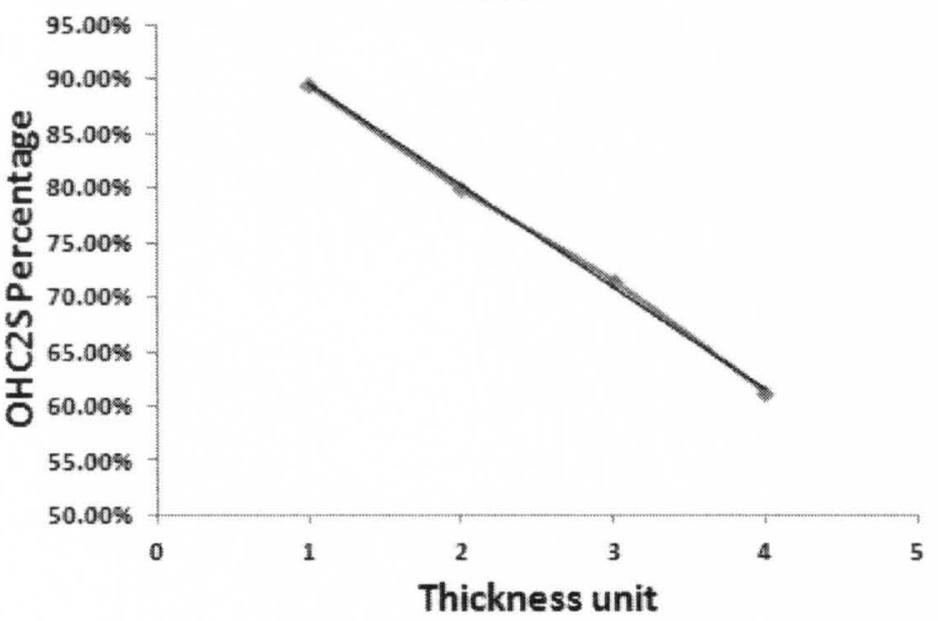

Figure 3.6. A) NMR spectra for 4 different MPC films of different thickness after a 4

h exchange with OHC2S: S1 is thinnest, S2 is double S1, S3 is triple, and S4 is fourfold in film thickness. B) The OHC2S percentage after $4 \mathrm{~h}$ exchange as a function of relative thickness determined by NMR. 


\subsubsection{Chemiresistive Sensing Response Towards IPA and Toluene.}

Figure 3.7. shows examples of chemiresistive sensing of IPA (solid plot) and Toluene (dashed plot). Figure 3.7A and 3.7B plot shows the sample after $0 \mathrm{~h}$ and $1 \mathrm{~h}$ vapor exchange, respectively. Figure $3.7 \mathrm{C}$ shows the response of the sensor after a $24 \mathrm{~h}$ exchange with OHC2S. The arrow indicates the time that the vapor was turned on and off. On corresponds to the vapor at $52 \%$ saturation with $\mathrm{N}_{2}$ and off correspond to $100 \%$ pure $\mathrm{N}_{2}$. In the presence of the vapor, the film of MPCs expands as the vapor molecule partitions into the film, increasing the cluster-cluster distance and reducing the film conductivity. The y-axis is the current passing through the device (I) divided by the initial baseline current $\left(\mathrm{I}_{\mathrm{b}}\right)$. This shows the relative change in conductivity in the presence of the vapor. Before exchange, the current decreases by $9 \pm 1 \%$ for IPA and $42 \pm 1 \%$ for the Toluene. The response is large for the non-polar toluene relative to IPA because the film is non-polar with all C6S ligands. ${ }^{1}$ After the 1 hour exchange, $24 \%$ of the $\mathrm{C} 6 \mathrm{~S}$ ligands were replaced by $\mathrm{OHC} 2 \mathrm{~S}$, leading to a current response to toluene of $33 \pm 3 \%$ and response to IPA to $10-15 \%$. The response is still larger for toluene. After 24 hours of exchange, $73 \%$ of the C6S ligands were replaced by OHC2S, making the film more sensitive to hydrophilic vapors. In this case, the response to IPA $(25-30 \%)$ was bigger than toluene (15\%). Figure 3.8. shows the response ratio $\left(\mathrm{R}_{I P A} / \mathrm{R}_{\text {Toluene }}\right)$ versus percentage of OHC2S in the film as determined by FTIR. The sensitivity for IPA versus toluene increased with increasing OHC2S ligand percentage from $0.2 \pm 0.1$ to $1.9 \pm 0.2$ for the response ratio $\left(R_{I P A} / R_{\text {Toluene }}\right)$ before and after one day exchange. In additional to the $52 \%$ vapor concentration, 


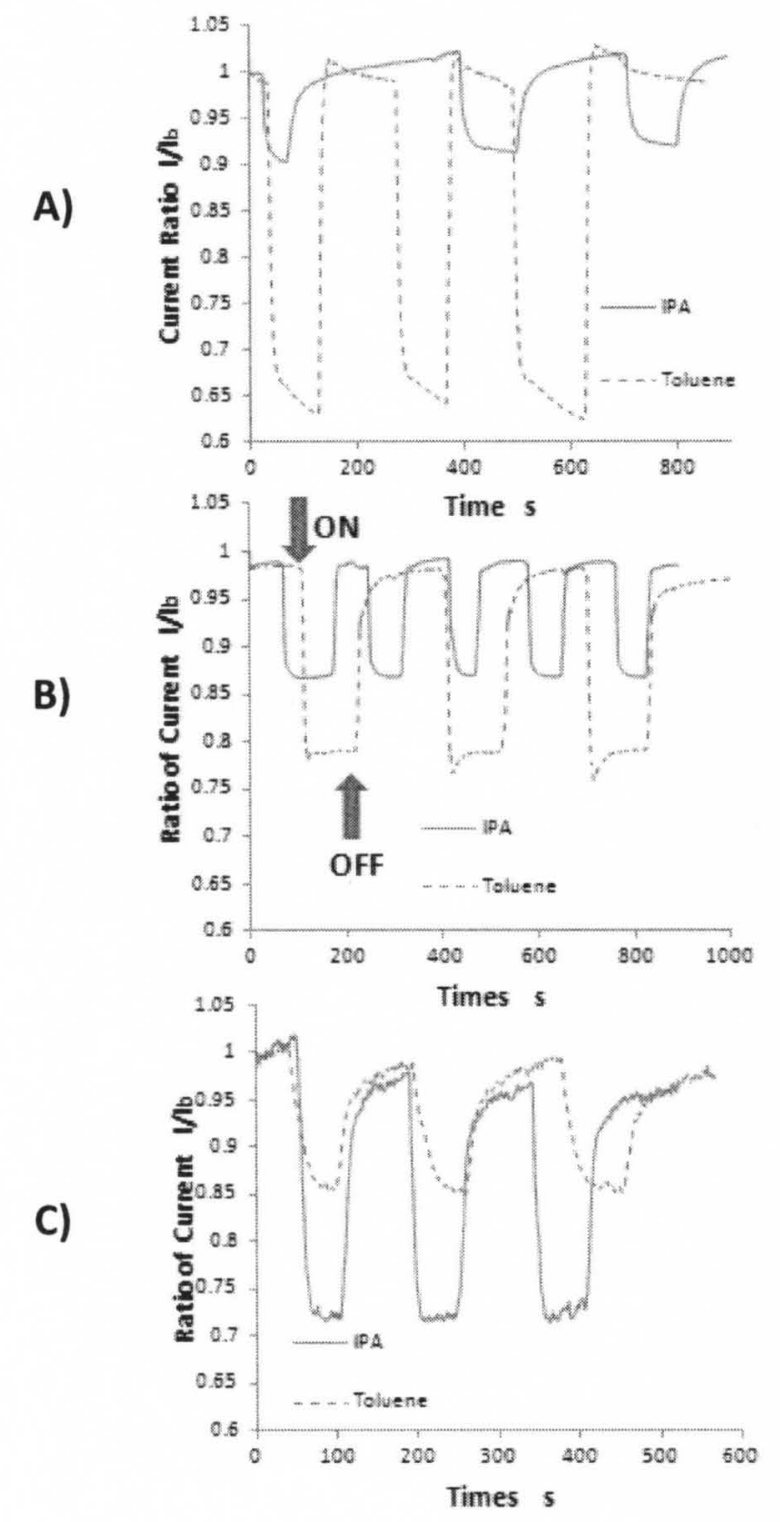

Figure 3.7. Chemiresistive sensing response of a film of C6S Au MPCs to 52\% IPA and toluene after exchange for A) $0 \mathrm{~h}, \mathrm{~B}) 1 \mathrm{~h}$, and C) $24 \mathrm{~h}$. The $\mathrm{ON}$ and OFF arrows indicate when the sensor is exposed to the vapor $(\mathrm{ON})$ and $\mathrm{N}_{2}$ only (OFF). 

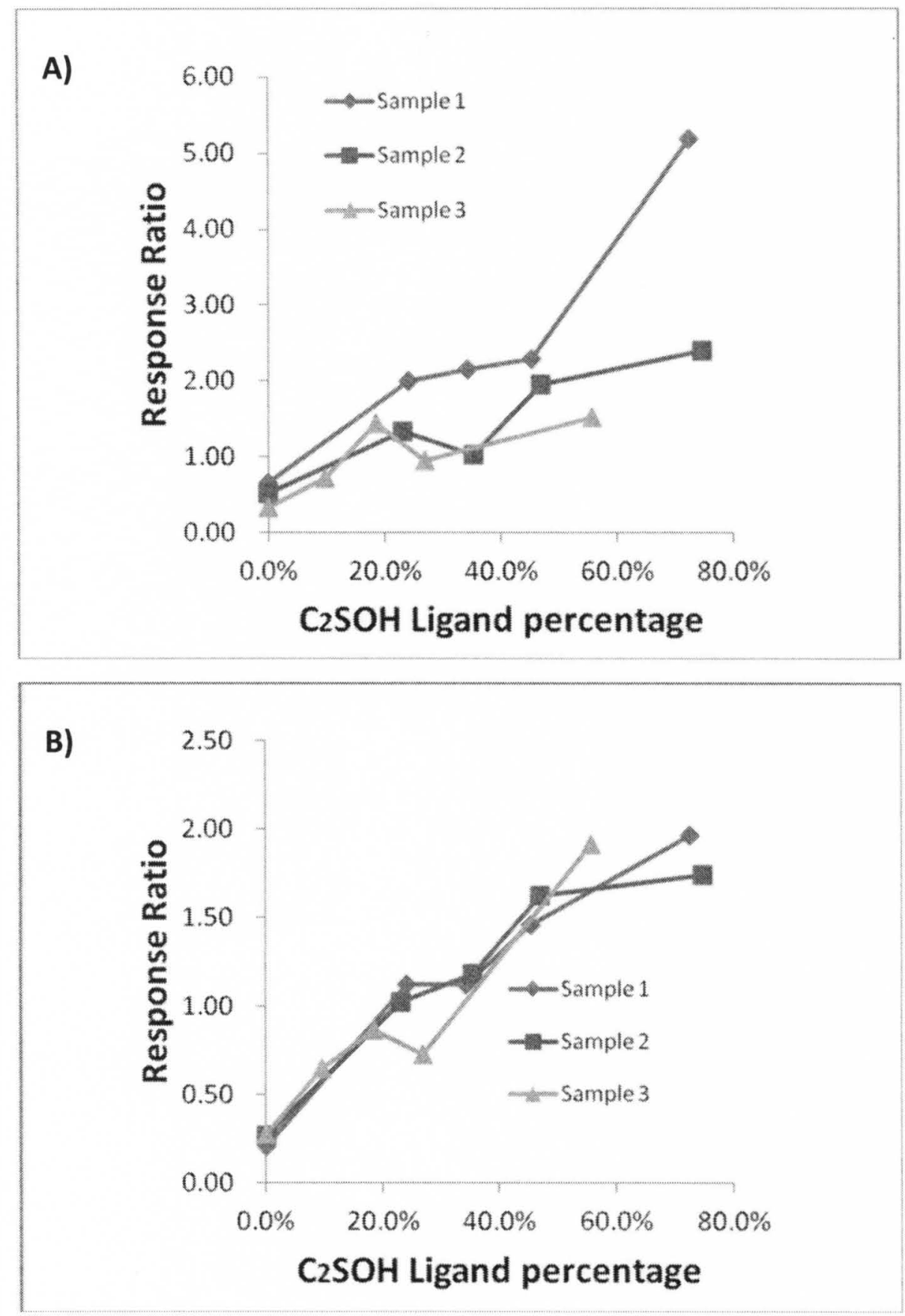

Figure 3.8. Ratio of the average current response (IPA to toluene) as a function of the percentage of $\mathrm{OHC} 2 \mathrm{~S}$ ligands on the $\mathrm{Au}$ MPCs for A) $4 \%$ and B) $52 \%$ concentration of IPA or Toluene. 
we also tested the sensitivity to $4 \%$ vapor concentration the same way. Again, the sensitivity to IPA increased with increasing $\mathrm{OHC} 2 \mathrm{~S}$ ligands. Figure $3.8 \mathrm{~A}$ shows the response ratio for the $4 \%$ vapor concentration. The variation in the response ratio as a function of OHC2S ligands is larger for the $4 \%$ concentration, but the trend is the same.

\subsection{Conclusions}

In this paper we described the use of a vapor phase place-exchange reaction to quickly vary the functionality of films of Au MPCs. FTIR and NMR spectra showed that the reaction was effective. After exchange, the new OHC2S ligands replaced the original $\mathrm{C} 6 \mathrm{~S}$ ligands. The exchange rate was relatively fast at early times and proceeded slower with increasing time, until it reached equilibrium near $90 \%$ exchange. After one day of exchange, $\sim 90 \%$ of the ligands were replaced by $\mathrm{OHC} 2 \mathrm{~S}$. We also studied the chemiresistive sensing selectivity of the film before and after exchange, which further confirmed the replacement of the protecting ligands. Before exchange, the protecting ligands were C6S, which are non-polar and have a better affinity for non-polar vapors (TOL). After exchange, the ligands were replaced by the more polar $\mathrm{OHC} 2 \mathrm{~S}$ and the sensitivity for polar vapors (IPA) increased. The chemiresistive sensing study indicated that the vapor phase place-exchange reaction could be utilized for VOC sensors and sensor arrays using metallic monolayer protected clusters (MPCs). 


\section{CHAPTER IV}

\section{CREATING SENSOR ARRAYS FOR VOLATILE ORGANIC COMPOUNDS USING VAPOR PHASE PLACE EXCHANGE ON GOLD MONOLAYER-PROTECTED CLUSTERS}

\subsection{Introduction}

Sensor array technology ${ }^{48}$ has gained a great deal of attention due to its unique ability to detect and analyze multi-component samples, which is difficult for single sensor systems to achieve. "Electronic nose"48-54 is the name given to sensor devices that are based on sensor array technology and are able to identify and quantify the vapor phase analytes in a complex mixture through pattern recognition methods. ${ }^{49-51}$ It has a wide range of applications in food analysis, ${ }^{74,75}$ clinical diagnostics, ${ }^{5}$ and environmental protection. ${ }^{43,44}$ Metal oxides, ${ }^{82-84}$ such as tin oxide, ${ }^{100}$ are the most popular sensor material for those applications because they are stable, robust, and sensitive to analytes at very low concentration. Several draw backs exist though, such as the need to operate at high temperature and the poor selectivity. Other materials that can be used as the sensor transducers are carbon nanotubes, ${ }^{95-97}$ carbon black/polymer composites, ${ }^{85,86,98}$ and Au monolayer protected clusters (MPCs). ${ }^{87-90,99}$ Chemical selectivity can be controlled in these materials by choosing the proper 
polymer matrices or protecting group on the Au MPCs. ${ }^{46,47}$

Electron conductivity through films of Au MPCs occurs by an electron hopping mechanism, ${ }^{28-30}$ where the conductivity depends on the distance between the edges of the metal cores in the film and the dielectric properties of the surrounding environment. When the cluster film is exposed to the vapor analytes, the interaction between the film and the analytes can potentially alter both variables. The analyte induced swelling increases the cluster distance, leading to increased resistance. At the same time, the dielectric property changes can also affect the resistance of the film. A larger dielectric leads to larger conductivity due to reduced burrier to electron hopping. ${ }^{28,29}$ As a result, the conductivity changes generate a signal for the analyte. For example, films of the n-octanethiol (OT) protected Au MPCs have been reported for the detection of toluene vapor at a few ppm concentration. ${ }^{91}$ However, the sensitivity of the film towards polar vapors was much lower than nonpolar vapors. In another example, Au MPCs with $\mathrm{OH}$ groups as a stabilizer showed better selectivity towards polar analytes, showing that Au MPCs could somewhat differentiate the analyte based on polarity. ${ }^{46,92-94}$

In order to better distinguish analytes not only by their polarities, Au MPCs with multiple functionalities can be used in a sensor array. The combined response of several sensors in an array offers the ability to distinguish different analytes. Currently, the most popular method to synthesize Au MPCs with different functionality is by synthesizing a new batch of $\mathrm{Au}$ MPCs for each sensor or through place exchange reactions. In place exchange reactions, the new functionality is incorporated onto the 
metal MPCs by exchange with the original ligands as discussed in Chapter 1. Conventionally, this reaction is performed in the solution phase. It has many draw backs. First, the functionalities used are limited by solubility issues. Second, it takes a few days to reach equilibrium. Third, the synthesis requires rotor evaporation, filtration, and rinsing procedures. Here we use a novel method to exchange the protecting ligands in the vapor phase, which successfully overcomes these disadvantages. Through vapor phase place exchange reactions, we build sensor arrays easily and effectively. The procedure could easily be used for mass production. In this study, we synthesized C6S Au MPCs and exchange films with mercaptopropyltrimethoxysilane (MPTMS), mercaptoethanol (OHC2S), and mercaptopropionic acid (MPA) to build an electronic nose that consists of sensor arrays made by those four types of sensors for detecting toluene (TOL), isopropanol (IPA), ethanol (EtOH) and acetone (ACT) vapors.

\subsection{Experimental}

4.2.1. Chemicals: Hexanethiol (HT, 96\%), mercaptoethanol (ME, 99\%), sodium borohydride (99\%), tetraoctylamonium bromide (TOABr, 98\%), toluene (Tl, 99.9\%), isopropanol (IPA, 99.9\%) mercaptopropionic acid (MPA, 98\%), and mercaptopropyltrimethoxysilane (MPTMS, 98\%) were purchased from commercial sources and used as received. Hydrogen tetrachloroaurate $\left(\mathrm{HAuCl}_{4} \cdot 3 \mathrm{H}_{2} \mathrm{O}\right)$ was synthesized from metallic Au. Barnstead Nanopure water $(\mathrm{R} \geq 17.8 \mathrm{M} \Omega-\mathrm{cm})$ was employed for all aqueous solutions. 
4.2.2. Synthesis: Hexanethiolate-coated gold monolayer-protected clusters (C6S Au MPCs) were synthesized according to the Brust reaction. Refer to previous description in Section 2.1. Chapter II.

4.2.3. Vapor exchange setup: The vapor exchange setup is the same as that described in Chapter II. In this case we put MPTMS or MPA in the center of the peti dish and a $\mathrm{KBr}$ salt plate and Au electrodes (with $23 \mu \mathrm{m}$ gap) with a film of drop-cast C6S Au MPCs was placed beside the thiol in the petri dish together. We made sure that the film thickness was approximately the same for all the samples by drop-casting 2 drops from a $\sim 20 \mathrm{mg} / \mathrm{mL}$ toluene solution of C6S Au MPCs. The electrode was later removed for vapor sensing and the salt plate removed for FTIR experiments at various times to monitor the exchange kinetics.

4.2.4. Vapor sensing experiment: Refer to previous description in Chapter II on page 24 .

\subsection{Results}

\subsubsection{Vapor Phase Place-Exchange Reactions for MPA and MPTMS on C6S}

Au MPCs. Figure 4.1. is the scheme for the exchange reaction of the thiols used in this study. Figure 4.2. shows FTIR spectra of films of C6S Au MPCs after vapor phase place exchange with MPTMS and MPA for various times. Fig 4.2A shows a series of FTIR spectra of a film of C6S Au MPCs after 0, 4, 12, 24 and 48 hours of exchange with MPA. The peak at $1700 \mathrm{~cm}^{-1}$ corresponds to the $\mathrm{C}=\mathrm{O}$ stretch of the MPA ligand and the peak at $2956 \mathrm{~cm}^{-1}$ corresponds to the asymmetric $\mathrm{CH}_{3}$ 


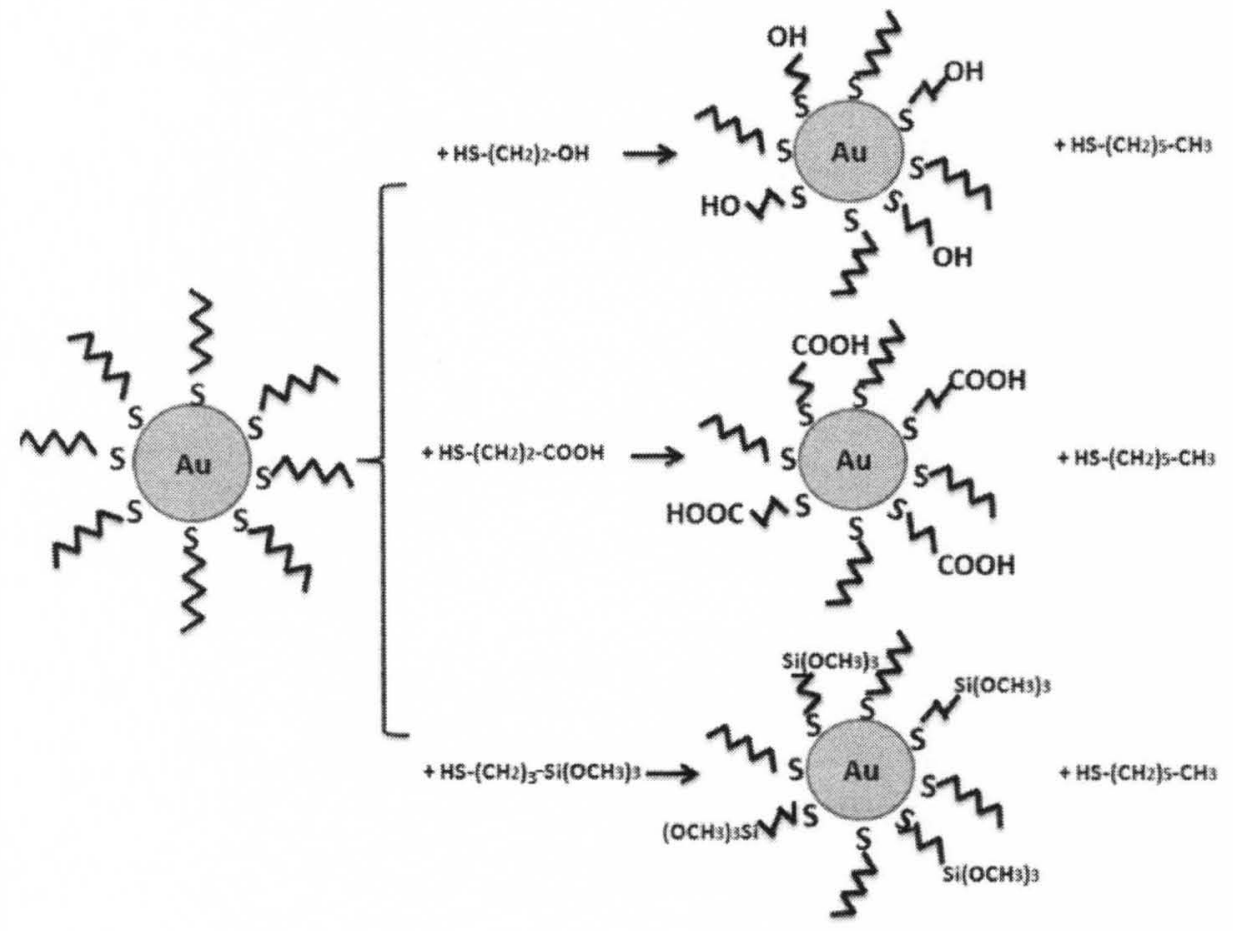

Figure 4.1. The scheme of exchange reactions for the three thiols, OHC2S, MPA, and MPTMS. 

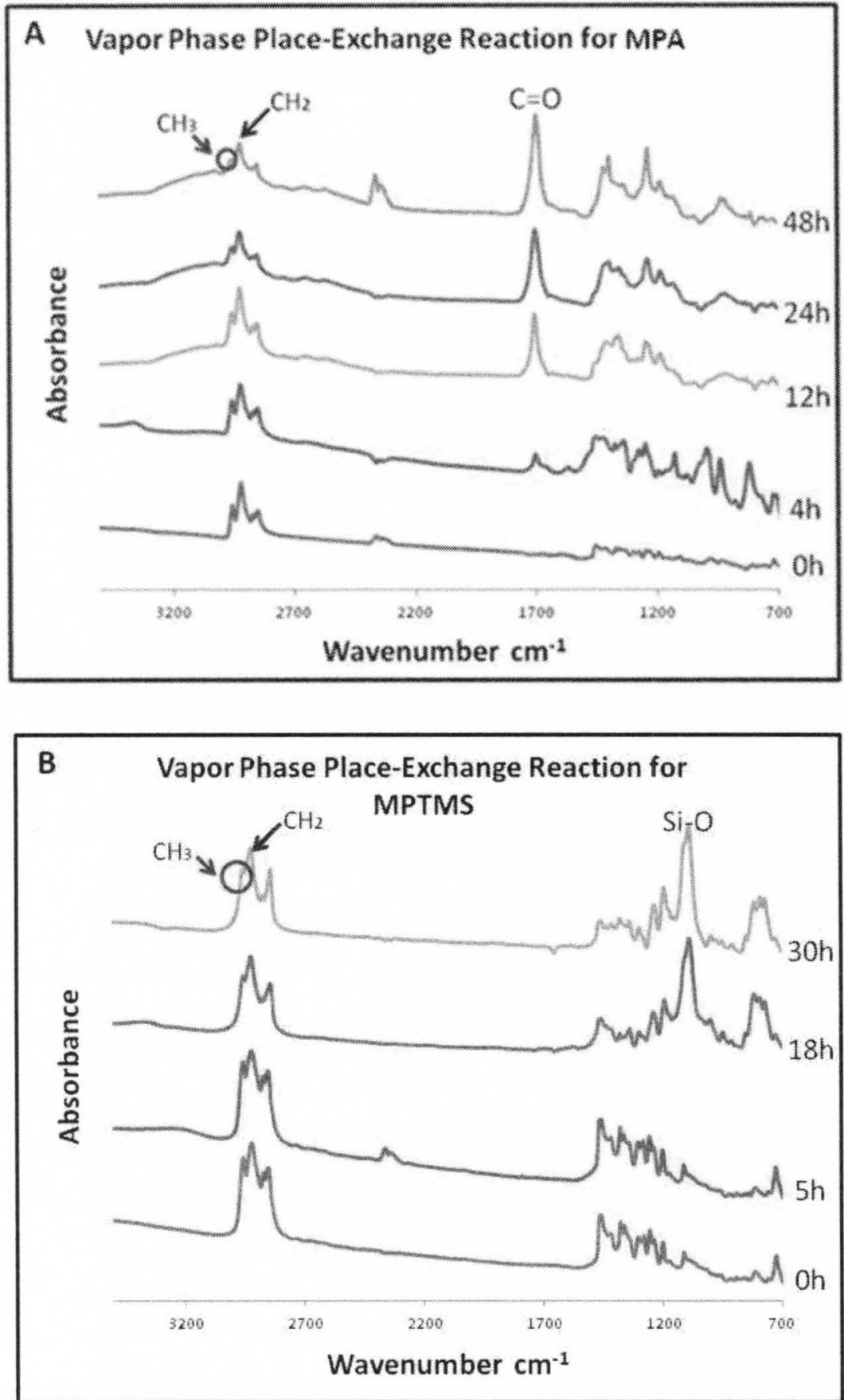

Figure 4.2. FTIR spectra of films of C6S coated Au MPCs exchanged with MPA (A) and MPTMS (B) in the vapor phase for various times as indicated. 
stretch of the $\mathrm{C} 6 \mathrm{~S}$ ligand. It is a shoulder peak of the asymmetric $\mathrm{CH}_{2}$ stretch at 2920 $\mathrm{cm}^{-1}$. The FTIR spectra show that the $\mathrm{C}=\mathrm{O}$ stretch increased in intensity with exchange time while the $\mathrm{CH}_{3}$ stretch intensity decreased, as expected for exchange of C6S ligands with MPA. Fig 4.2B shows a series of FTIR spectra for the film of C6S Au MPCs after 0, 5, 18 and 30 hours of exchange with MPTMS. The peak at 1100 $\mathrm{cm}^{-1}$ corresponds to the Si-O asymmetric stretch of the MPTMS. The peak at 2956 $\mathrm{cm}^{-1}$ corresponds to the asymmetric $\mathrm{CH}_{3}$ stretch of the $\mathrm{C} 6 \mathrm{~S}$ ligand and the peak at $2920 \mathrm{~cm}^{-1}$ corresponds to the asymmetric $\mathrm{CH}_{2}$ stretch. These two peaks decreased with increasing exchange time as expected for loss of the C6S ligands in both exchange reactions. We conclude that original protecting ligands were replaced by MPA and MPTMS in both cases. The FTIR spectra showed that for the MPTMS exchange after about one day, the methyl group significantly decreased in intensity. We attribute this to the hydrolysis reaction on the $\mathrm{Si}-\mathrm{O}-\mathrm{CH}_{3}$ group in air, which leads to the formation of $\mathrm{Si}-\mathrm{OH}$ or $\mathrm{Si}-\mathrm{O}-\mathrm{Si}$ groups in the film. Figure 4.3. shows the percentage of vapor exchange for MPA and MPTMS, which is very roughly approximated by peak height comparisons of the $\mathrm{CH}_{3}$, $\mathrm{Si}-\mathrm{O}$, and $\mathrm{C}=\mathrm{O}$ peak heights at different exchange times.

\subsubsection{Chemiresistive Vapor Sensing and Response Patten Generated by a}

Au MPC Electronic Nose. Figure 4.4 to Figure 4.7 shows a series of chemiresistive sensing response of $\mathrm{C} 6 \mathrm{~S} \mathrm{Au}, \mathrm{C} 6 \mathrm{~S} / \mathrm{OHC} 2 \mathrm{~S} \mathrm{Au}, \mathrm{C} 6 \mathrm{~S} / \mathrm{MPA} \mathrm{Au}$, and C6S/MPTMS Au to 4 different volatile organic compounds (VOCs). The 4 sensors are Au electrode 


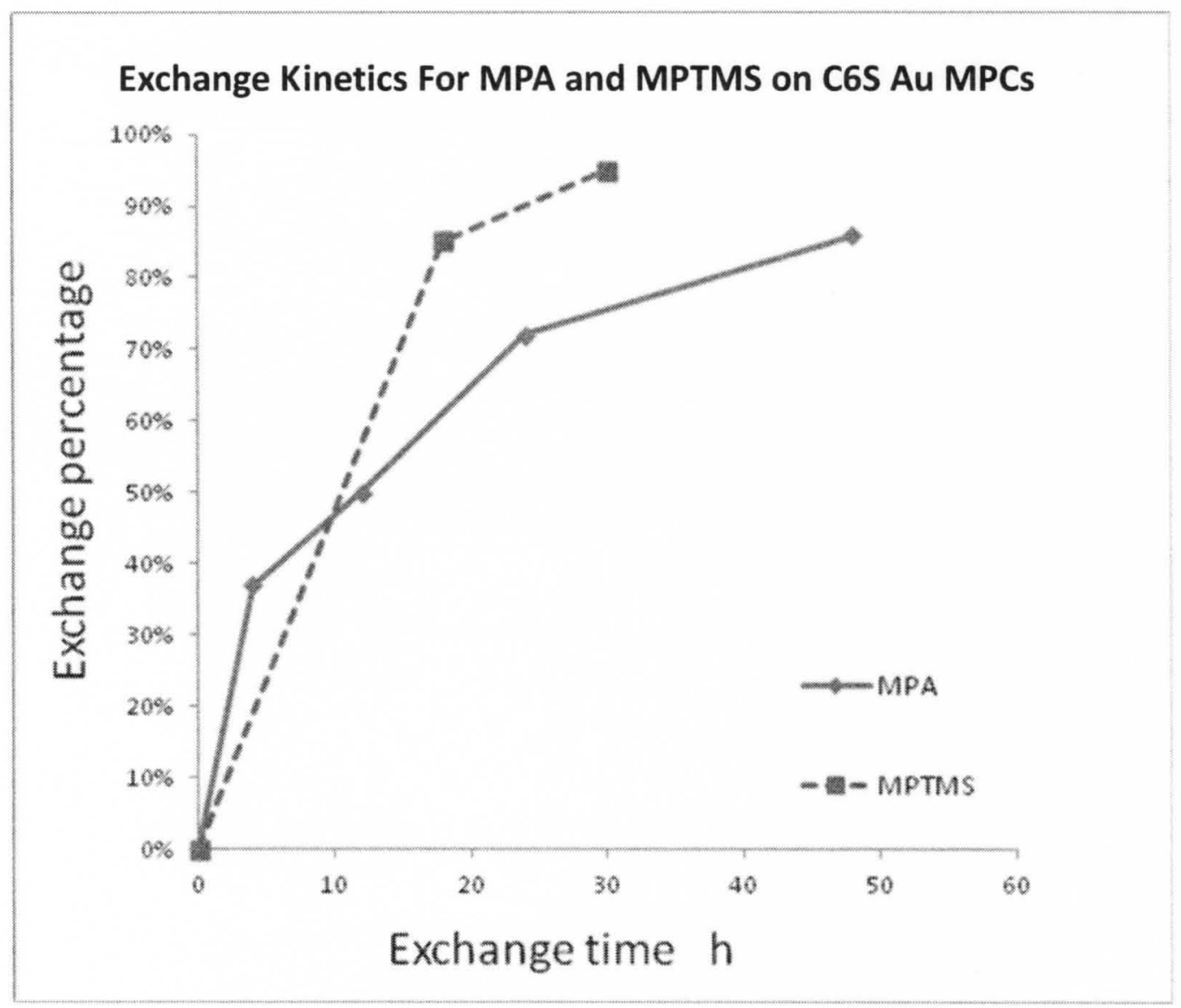

Figure 4.3. Approximation of the exchange kinetics for exchange of C6S Au MPC with MPA and MPTMS, calculated based on the FTIR peak height of a functional group of the ligands. 
devices coated with films of C6S Au MPCs and films of C6S Au MPCs after exchange with OHC2S, MPA, MPTMS thiols for 24 hours, where the percentage of ligand exchange was $78 \%, 85 \%$, and $87 \%$, respectively, estimated by the peak height of the appropriate functional end group. Figure 4.4. shows the sensing responses of the four sensors to toluene vapor. The signal was obtained by measuring the current change while holding the potential constant at $0.3 \mathrm{~V}$. We plotted the current versus time in the figure. The down arrow indicates when the device was exposed to the VOC $/ \mathrm{N}_{2}$ mixture at $52 \%$ and the up arrow indicates when the device was exposed to pure $\mathrm{N}_{2}$ gas. The response is calculated as follows:

$$
\text { Response }=\Delta \mathrm{i} / \mathrm{i}_{\mathrm{b}}=\left|\mathrm{i}_{\mathrm{b}}-\mathrm{i}_{\mathrm{r}}\right| \mathrm{i}_{\mathrm{b}}
$$

where $i_{b}$ is background current in the presence of pure $N_{2}$ and $i_{r}$ is the current when the device is exposed to the VOC/ $\mathrm{N}_{2}$ mixture.

The four plots in Figure 4.4. show that films of C6S and MPTMS Au MPCs have greater sensitivity to toluene compared to films of OHC2S and MPA Au MPCs. This is likely due to the more nonpolar nature of those films. The response time and recovery time also varied. Films of OHC2S and MPA Au MPCs exhibited a $30 \mathrm{~s}$ response and recovery time, while the C6S and MPTMS Au MPCs responded and recovered within 5 seconds. Figure 4.5 shows the sensing response of the devices to IPA vapor. IPA is a more polar analyte and therefore the OHC2S showed a larger response compared to C6S. Interestingly, the MPTMS Au MPCs also responded well to IPA. The C6S sensor had the worst sensitivity to IPA vapor due to the opposite polarity between the coating ligands and 


\section{TOLUENE}
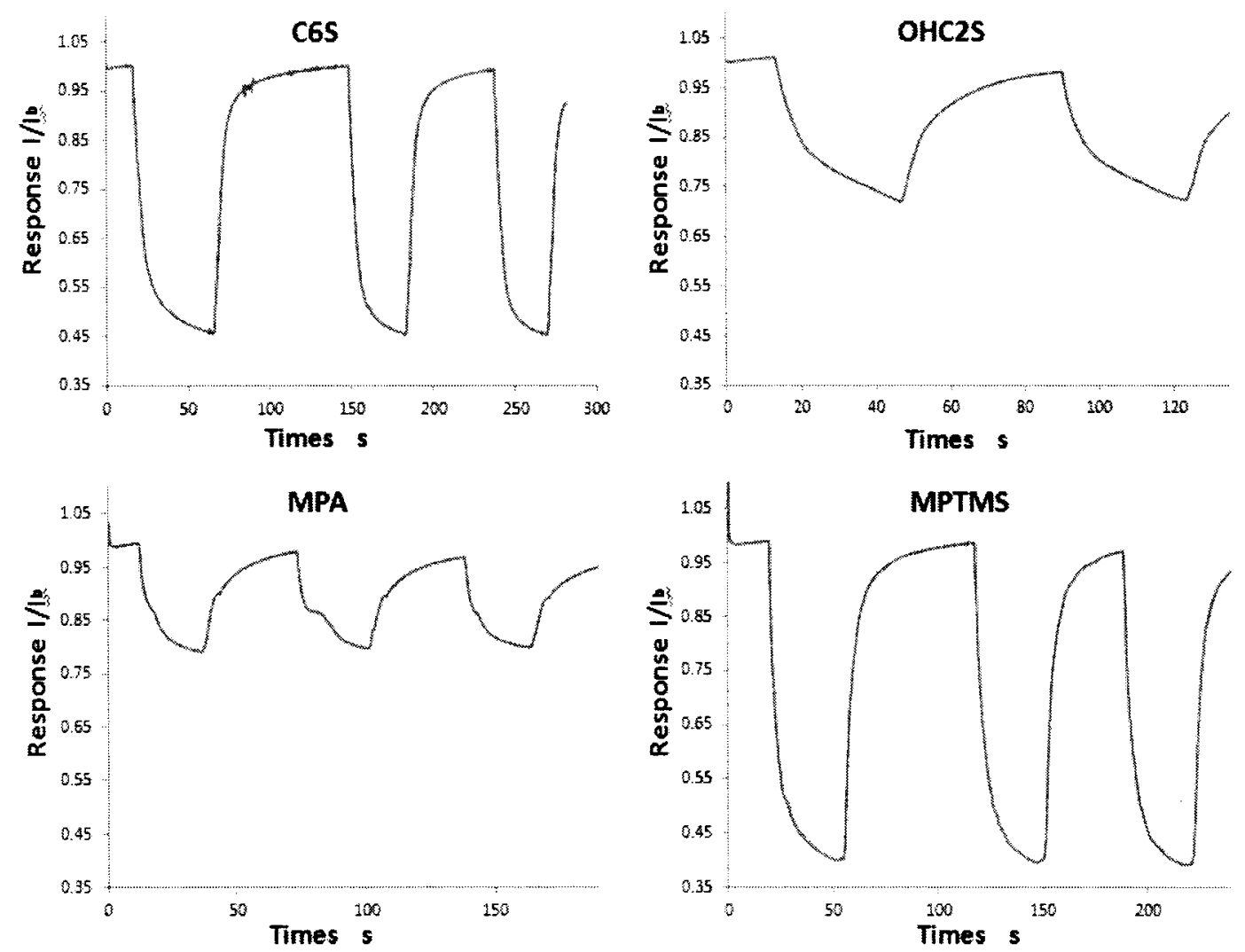

Figure 4.4. The chemiresistive sensing response to toluene vapor at $52 \%$ saturation, generated by 4 types of sensors made by a $24 \mathrm{~h}$ vapor phase exchange: C6S Au MPCs, OHC2S Au MPCs, MPA Au MPCs, and MPTMS Au MPCs. 


\section{IPA}
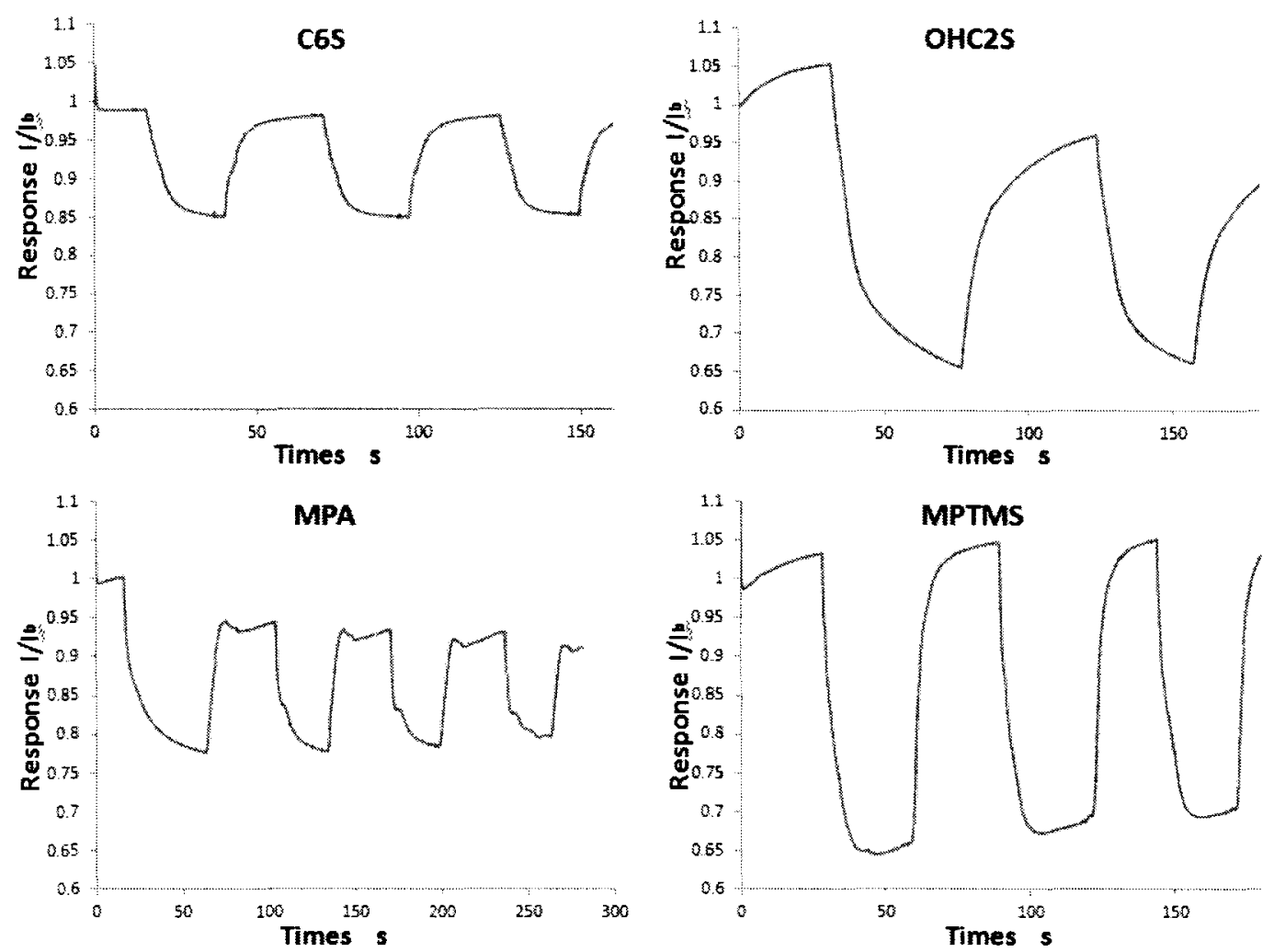

Figure 4.5. The chemiresistive sensing response to IPA vapor at $52 \%$ saturation, generated by 4 types of sensors made by $24 \mathrm{~h}$ vapor phase exchange: C6S Au MPCs, OHC2S Au MPCs, MPA Au MPCs, and MPTMS Au MPCs. 
ETHANOL
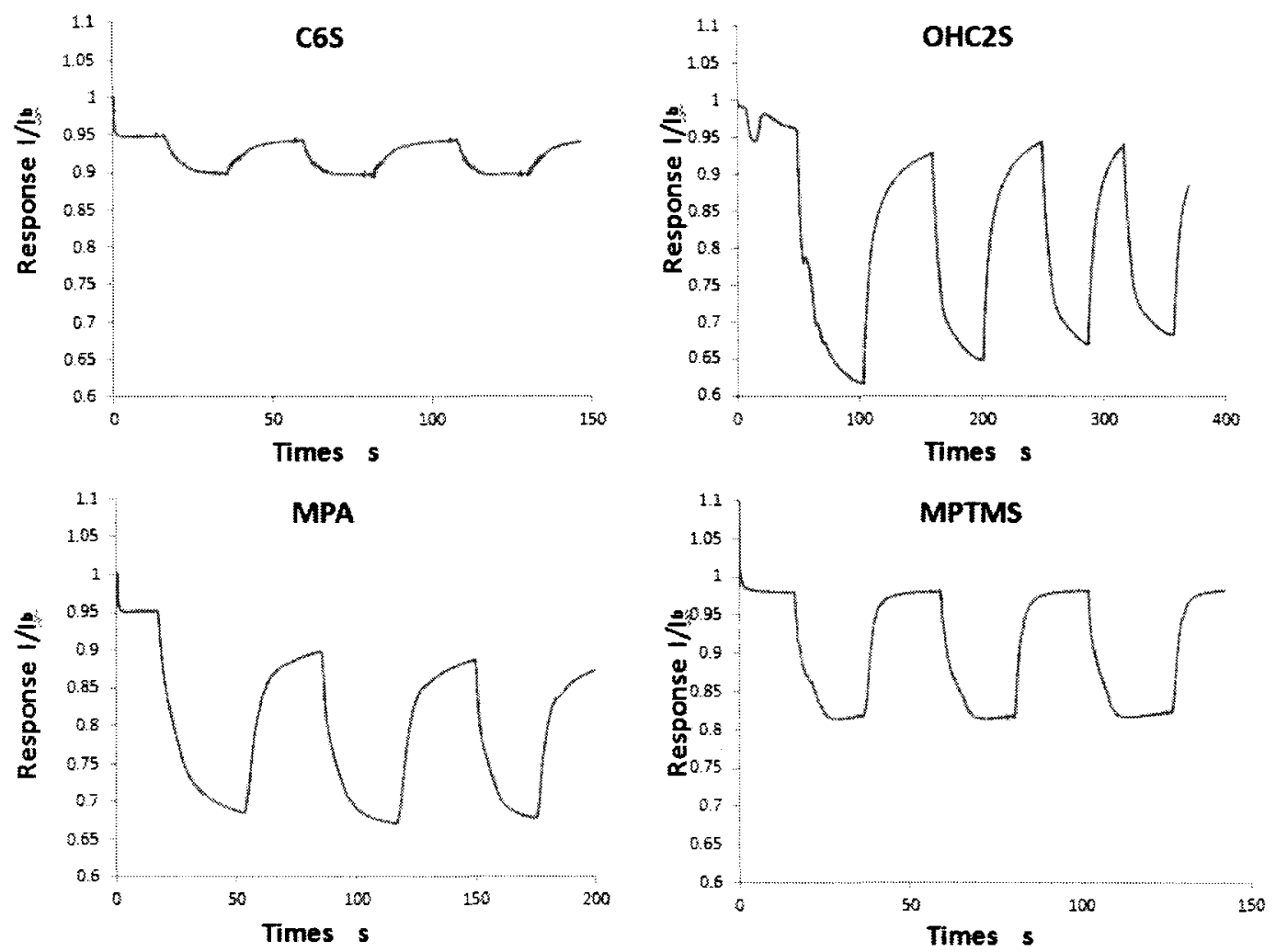

Figure 4.6. The chemiresistive sensing response to ethanol vapor at $52 \%$ saturation, generated by 4 types of sensors made by $24 \mathrm{~h}$ vapor phase exchange: C6S Au MPCs, OHC2S Au MPCs, MPA Au MPCs, and MPTMS Au MPCs. 


\section{ACETONE}
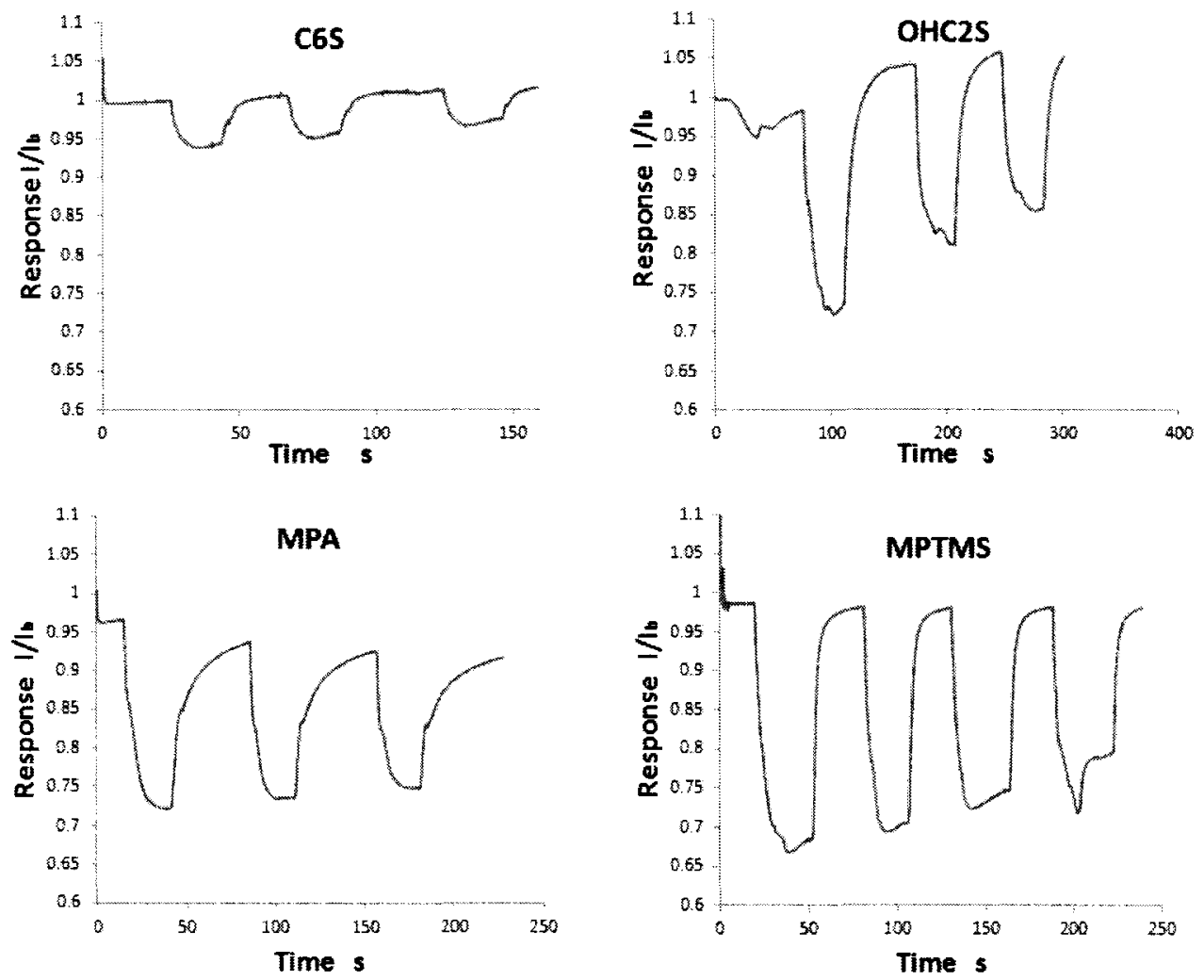

Figure 4.7. The chemiresistive sensing response to acetone vapor at $52 \%$ saturation, generated by 4 types of sensors made by 24 h vapor phase exchange: C6S Au MPCs, OHC2S Au MPCs, MPA Au MPCs, and MPTMS Au MPCs. 
the vapor. The response and recovery time was faster for C6S, MPA and MPTMS Au MPC films compared to OHC2S. Figure 4.6. shows the sensing response for ethanol. The C6S Au MPCs again gave the smallest response due to the mismatch polarity. The response was $\Delta \mathrm{I} / \mathrm{I}_{0} \approx 5 \%$ to ethanol compared to $13 \%$ for IPA, because ethanol is more polar than IPA. The response time and recovery time of the C6S Au MPC film also increased for the polar analyte ethanol. Figure 4.7 shows the responses to acetone vapor. The responses of OHC2S, MPA and MPTMS Au MPCs were significantly larger than the C6S Au MPCs likely due to the hydrogen bonding interaction between the analytes and the ligands and better match in polarity for the former. All responses for the different sensors and analyte are shown in Table 4.1.

4.3.3 Response Pattern Generated by The Electronic Nose. Figure 4.8. shows the sensing response pattern of the 4 VOCs generated by the electronic nose comprised of the Au electrode devices with films of C6S, OHC2S, MPA, and MPTMS Au MPCs. It is clear that each analyte has a unique response pattern consisting of 4 descriptors generated by the 4 types of sensors. Those patterns can be considered as "finger prints" for the specific vapors. It is possible to identify the vapors even in a mixture of gas by pattern recognition methods such as principal component analysis.

\subsection{Conclusions}

In this project we successfully altered the functionalization of films of Au MPCs through vapor phase place exchange reactions. In addition to our previous work on the 
Table 4.1. Chemiresistive sensing response to VOCs by the list of sensors below.

\begin{tabular}{|c|c|c|c|c|}
\hline VOCs & Toluene & IPA & Ethanol & Acetone \\
\hline C6S & $54.6 \% \pm 0.6 \%$ & $13.2 \% \pm 0.2 \%$ & $4.9 \% \pm 0.2 \%$ & $3.5 \% \pm 0.2 \%$ \\
\hline OHC2S & $27.3 \% \pm 0.6 \%$ & $32.3 \% \pm 1.5 \%$ & $28.7 \% \pm 1.5 \%$ & $22.9 \% \pm 1.5 \%$ \\
\hline MPA & $18.3 \% \pm 1.2 \%$ & $16.0 \% \pm 1.0 \%$ & $24 \% \pm 1.0 \%$ & $21.7 \% \pm 2.5 \%$ \\
\hline MPTMS & $59.3 \% \pm 0.6 \%$ & $34.3 \% \pm 1.5 \%$ & $16.1 \% \pm 0.3 \%$ & $27.3 \% \pm 3.1 \%$ \\
\hline
\end{tabular}




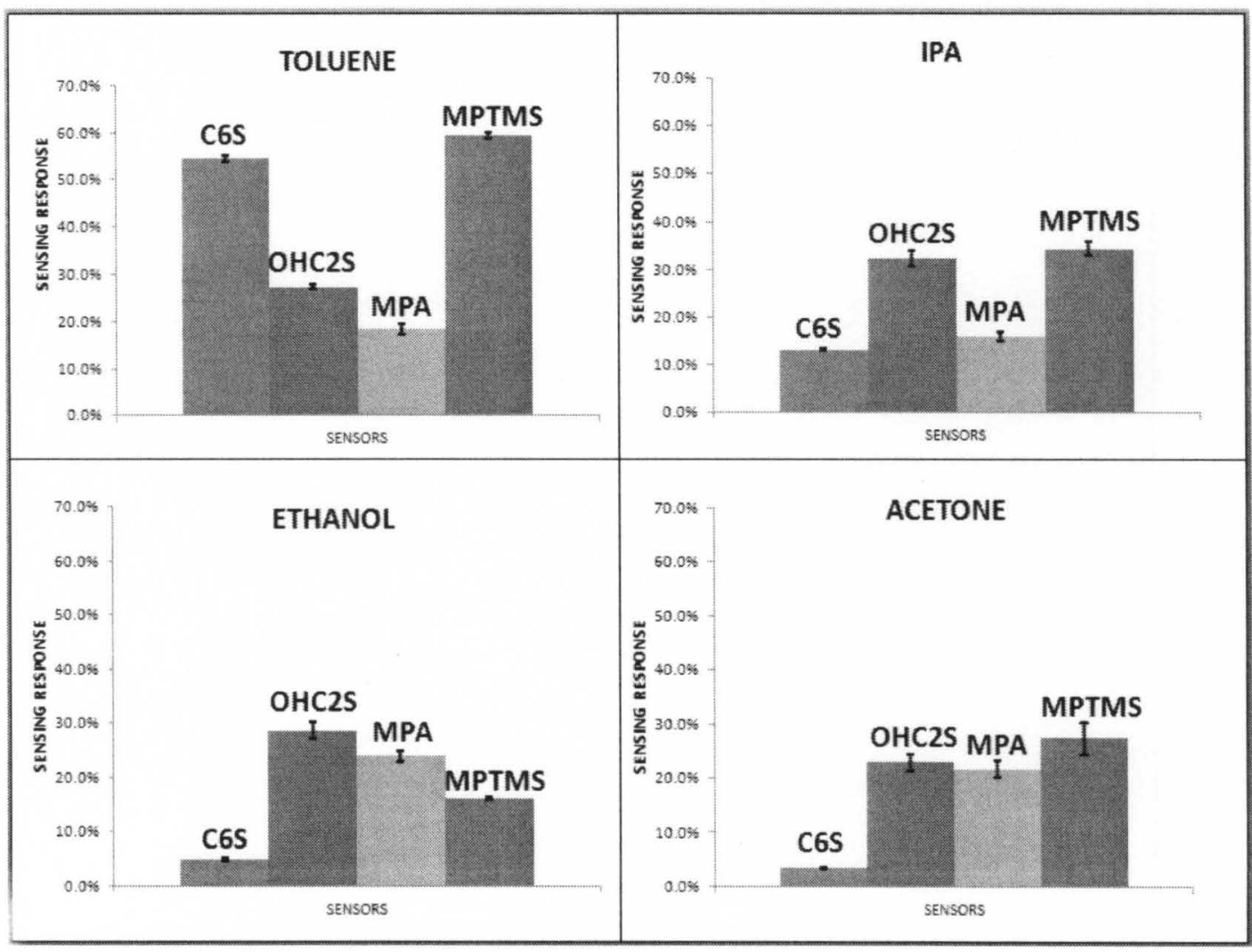

Figure 4.8. The response patterns of toluene, IPA, acetone and ethanol vapor provided by the chemiresistive electronic nose consisting of $\mathrm{Au}$ electrode devices coated with films of $\mathrm{C} 6 \mathrm{~S}, \mathrm{OHC} 2 \mathrm{~S}, \mathrm{MPA}$, and MPTMS Au MPCs. From left to right: C6S, OHC2S, MPA and MPTMS. 
synthesis of $\mathrm{Au}$ MPCs coated with $\mathrm{OHC} 2 \mathrm{~S}$ ligands through vapor phase place exchange, we further utilized the method to synthesize Au MPCs with mixed ligands of C6S and MPA or C6S and MPTMS, leading to 4 different types of Au MPC film. From the FTIR spectra we observe that the original ligand on the MPCs is partially replaced by the new ligands MPA or MPTMS. The ligand ratio is approximated by FTIR spectroscopy. We investigated applications as an electronic nose. The electronic nose was constructed from sensor arrays consisting of the four types of sensors made by drop cast films of C6S Au MPCs on the IDA electrodes followed by vapor exchange with OHC2S, MPA and MPTMS reagents. The chemiresistive sensing response patterns to toluene, IPA, ethanol and acetone were generated by the electronic nose and were distinguishable from each other. This study indicates that that vapor phase place exchange reactions can be used for quickly producing films of Au MPCs with various functionalities for constructing electronic nose device for VOC detection. while only 4 films were demonstrated here, much more functionalities could be rapidly created from one batch of C6S Au MPCs by vapor exchange using different thiols, different amounts of exchange, and using 3 or more different thiols in one film. This is much more convenient method for altering functionality compared to synthesizing a different batch of MPCs for each sensor, which commonly done. 


\section{CHAPTER V}

\section{SUMMARY AND FUTURE DIRECTIONS}

In this thesis we discussed the procedure, kinetics, and sensing applications of vapor phase place exchange reactions used for altering the functionalizations of films of $\mathrm{Au}$ MPCs. In the first chapter we introduced the history of Au MPCs, including synthesis, electronic properties, and chemiresistive sensing applications. $\mathrm{Au}$ nanoparticles with ionic stabilizers were first synthesized in the $1950 \mathrm{~s}^{9} \quad$ The method for synthesis is through citrate reduction and it is still popular for various applications.

On the other hand, Au MPCs, stabilized by organomercaptan groups, are more stable and can be isolated as a solid and dissolved repeatedly. They are synthesized by the Brust method, ${ }^{10}$ which is the method we used for preparing Au MPCs in this work. The properties of the Au MPCs largely depend on the protecting ligands. In order to synthesize multiple types of functionalized $\mathrm{Au}$ MPCs, researchers developed various methods, such as place exchange reactions and coupling reactions. The electronic properties of the Au MPC film can be described by an "electron hopping mechanism", where the conductivity of the MPC film depends on the distance between the Au cores and the dielectric properties of the surroundings. Au MPCs can be used for many applications, including sensors, energy, separations and catalysis. Our research focused on chemiresistive sensing applications for the detection of VOCs. The 
sensor selectivity depends on the functionality of the Au MPCs, while the sensitivity depends on the flexibility, film thickness, metal size, and ligand spacing. Electronic noses consisting of $\mathrm{Au}$ MPC sensors can be built for further distinguishing vapor analytes, especially in a mixture, as long as a sensor array can be fabricated

In Chapter II, we introduced our experimental methods and instrumentation. First, we synthesize Au MPCs with hexanethiol as a monolayer stabilizer by the Brust method. MPC films were prepared by drop cast deposition from the MPC toluene solution. FTIR and NMR spectroscopy allowed MPC characterization and monitoring of the vapor phase place exchange kinetics. FTIR is a faster technique but not as accurate as NMR spectroscopy. The NMR spectroscopy has good sensitivity and could determine the ligand ratio accurately, but it took longer and suffered from solubility requirements. For our lab, we analyzed the MPC film in the solid state, which is easier to measure by FTIR.

In Chapter III we described the vapor phase place-exchange procedure to quickly vary the functionality of the films of C6S coated Au MPCs with OHC2S ligands. Based on FTIR and NMR measurements, up to 91 percent of the original C6S ligands became replaced by the new incoming $\mathrm{OHC} 2 \mathrm{~S}$ ligands in the vapor phase. In the beginning of the exchange, the reaction rate was relatively fast, and became slower with increasing time as the reaction reached equilibrium. Also, the reaction kinetics were slower and extent of exchange less for thicker Au MPC films. Our procedure allowed control over the amount of exchange easily by adjusting the exchange time. Through vapor phase thiol place exchange, we could significantly alter the vapor 
sensing selectivity towards isopropanol over toluene.

In Chapter IV we demonstrated vapor phase place exchange reactions for synthesizing Au MPCs with other functionalities, including MPTMS and MPA. The original C6S ligands on the Au MPCs can be replaced by both MPTMS and MPA in the vapor phase, We explored the application of vapor phase place exchange reactions for fabricating electronic noses that consist of four sensors with films of Au MPCs functionalized with 1) pure $\mathrm{C} 6 \mathrm{~S}$, 2) $\mathrm{C} 6 \mathrm{~S} / \mathrm{OHC} 2 \mathrm{~S}$, 3) C6S/MPA, 4) C6S/MPTMS. This sensor array allowed us to detect and distinguish between toluene, IPA, ethanol, and acetone. The sensor array produced unique chemiresistive sensing response patterns for each VOC. Pattern recognition method could be used to identify the vapors.

In the future we can explore the application of vapor phase place exchange reactions for synthesizing metal MPCs with various functionalities for sensing, catalysis or biosensing applications. There is also a need to study the detailed mechanism of exchange and the effect of temperature or film thickness on the exchange kinetics. We also want to explore the response of other ligands difficult to synthesize in solution, such as fluorinated thiols and other thiols to build larger sensor arrays to distinguish more analytes, applying pattern recognition software, and analyzing complex mixtures. Coupling reactions on the original ligands could be used as a secondary reaction to further vary functionality in the vapor phase place exchange experiments, A quantitative comparison of the kinetics of the two reactions could be another interesting project to explore. 


\section{REFERENCES}

[1] Wohltjen, H.; Snow, A. W. Anal. Chem. 1998, 70, 2856.

[2] Choi, J. P.; Coble, M. M.; Branham, M. R.; DeSimone, J. M.; Murray, R. W. J. Phys. Chem. C 2007, 111, 3778-3785

[3] Ibanez, FJ.; Gowrishetty, U.; Crain, MM.; Walsh, KM.; Zamborini, FP. Anal. Chem. 2006, 78, 753-761.

[4] Ibanez, FJ.; Zamborini, FP. ACS nano. 2008. 2, 1543-1552.

[5] Cao, W.; Duan, Y.; Clin. Chem. 2006, 52, 800-811.

[6] Gross, GM.; Grate, JW.; Synovec, RE.; J. Chromatogr. A. 2004, 1060, 225-236.

[7] Cai, QY.; Zellers, ET. Anal. Chem. 2002, 74, 3533-3539.

[8] Long, CG.; Gilbertson, JD.; Vijayaraghavan, G.; Stevenson, KJ.; Pursell, CJ.; Chandler, BD.; J.Am.Chem.Soc. 2008, 130, 10103-10115.

[9] Turkevitch, J.; Stevenson, P. C.; Hillier, J. Faraday Soc. 1951, 11, 55-75.

[10] Brust, M.; Walker, M.; Bethell, D.; Schiffrin, D. J.; Whyman, R. J. Chem. Soc., Chem. Commun. 1994, 7, 801-802.

[11] Frens, G. Nature: Phys. Sci. 1973, 241, 20-22.

[12] Yonezawa, T.; Kunitake, T.; Colloids Surf. A: Physicochem. Eng. Asp. 1999, $149,193-199$.

[13] Jana, N. R.; Gearheart, L.; Murphy, C. J. J. Phys. Chem. B 2001, 105, 4065. 
[14] Murphy, C. J.; Sau, T. K.; Gole, A. M.; Orendorff, C. J.; Gao, J.; Gou, L.; Hunyadi, S. E.; Li, T. J. Phys. Chem. B 2005, 109, 13857.

[15] Kaminker, R.; Lahav, M.; Motiei, L.; Vartanian, M.; Popovitz-Biro, R.; Iron, M. A.; M. E. van der Boom, Angew. Chem. Int. Ed. 2010, 49, 1218.

[16] Gittins, D. I.; Caruso, F. J. Phys. Chem. B 2001, 105, 6846.

[17] Giersig, M.; Mulvaney, P. Langmuir 1993, 9, 3408-3413.

[18] Brust, M.; Fink, J.; Bethell, D.; Schiffrin, D.J.; Kiely, C. J. J. Chem. Soc. Chem. Commun. 1995, 1655-1656.

[19] Hostetler, M. J.; Templeton, A. C.; Murray, R. W. Langmuir 1999, 15, 3782.

[20] Luo, J.; Kariuki, N.; Han, L.; Maye, M.M.; Moussa, L.W.; Kowaleski, S.R.; Kirk, F.L.; Hepel, M.; Zhong, C.J. J. Phys. Chem. B 2002, 106, 9313-9321

[21] Lover, T.; Henderson, W.; Bowmaker, G.A.; Seakins, J.M.; Cooney, R.P. Chem. Mater. 1997, 9, 1878-1886.

[22] Brown, L.O.; Hutchison, J.E. J. Am. Chem. Soc.1997, 119, 12384-12385.

[23] Shaffer, A.W.; Worden, J.G.; Huo, Q. Langmuir 2004, 20, 8343-8351.

[24] Jana, N. R.; Gearheart, L.; Murphy, C. J. J. Phys. Chem. B 2001, 105,4065 .

[25] Murphy, C. J.; Sau, T. K.; Gole, A. M.; Orendorff, C. J.; Gao, J.; Gou, L.; Hunyadi, S. E.; Li, T. J. Phys. Chem. B 2005, 109, 13857.

[26] Chailapaku, O.; Sun, L.; Xu, C.; Crooks, R.M. J. Am. Chem. Soc. $1993,115,12459-12467$.

[27] Briglin, S.M.; Gao, T.; Lewis, N.S. Langmuir 2004, 20, 299-305. 
[28] Terrill, R. H.; Postlethwaite, T. A.; Chen, C-H.; Poon, C-D.; Terzis, A.; Chen, A.; Hutchison, J.E.; Clark, M.R.; Wignall, G.; Londono, J.D. J. Am. Chem. Soc. $1995,117,12537-12548$.

[29] Wuelfing, W.P.; Green, S.J.; Pietron, J.J.; Cliffel, D.E.; Murray. R.W. J. Am. Chem. Soc.2000, 122, 11465-11472.

[30] Zamborini, F.P.; Smart, L.E.; Leopold, M.C.; Murray, R.W. Anal. Chim. Acta 2003, 496, 3-16.

[31] Sheng, P.; Abeles, B. Phys. Rev. Lett. 1972, 28, 34.

[32] Terrill, R. H.; Postlethwaite, T. A.; Chen, C.; Poon, C.; Terzis, A.; Chen, A.; Hutchison, J. E.; Clark, M. R.; Wignall, G.; Londono, J. D.; Superfine, R.; Falvo, M.; Johnson, C. S., Jr.; Samulski, E. T.; Murray, R. W. J. Am. Chem. Soc. 1995, 117, 12537.

[33] Franke, M. E.; Koplin, T. J.; Simon, U. Small 2006, 2, 36.

[34] Albert, K. J.; Lewis, N. S.; Schauer, C. L.; Sotzing, G. A.; Stitzel, S. E.; Vaid, T. P.; Walt, D. R. Chem. Rev. 2000, 100, 2595.

[35] Dutta, R.; Hines, E. L.; Gardner, J.W.; Kashwan, K. R.; Bhuyan, M. Sens. Actuators B 2003, 94, 228.

[36] Yan, H.; Choe, H.; Nam, S. S.; Hu, Y.; Das, S.; Klemic, J. F.; Ellenbogen, J. C.; Lieber, C. M. Nature 2010, 470, 240.

[37] Hollidey, B. J.; Stanford, T. B.; Swager, T.M. Chem.Mater. 2006, 18, 5649.

[38] Lewis, N. S. Acc. Chem. Res. 2004, 37, 663.

[39] Lewis, F. A. The Palladium/Hydrogen System, Academic Press Inc., 
London 1967.

[40] Favier, F.; Walter, E.C.; Zach, M. P.; Benter, T. R.; Penner, M. Science 2001, $293,2227$.

[41] Mitsubayashi, K.; Yokoyama, K.; Takeuchi, T.; Karube, I. Anal. Chem. 1994, $66,3297-3302$.

[42] Strike, D. J.; Meijerink, M. G. H.; Koudelka-Hep, M. J. Anal. Chem. 1999, $364,499-505$.

[43] Finlayson-Pitts, B.; Pitts, J. N. Science 1997, 276, 1045-1052.

[44] Pejcic, B.; Eadington, P.; Ross, A. Crit. Rev. 2007, 41, 6333-6342.

[45] Fitch, J. P.; Raber, E.; Imbro, D. R. Science 2003, 302, 1350-1354.

[46] H.-L. Zhang, S. D.; Evans, J. R.; Henderson, R. E.; Miles, T.-H. Nanotechnology 2002, 13, 439.

[47] Evans, S.D.; Johnson, S.R.; Cheng, Y.L.; Shen, T. J. Mater. Chem. 2000, 10, 183.

[48] Persaud, K.C.; Dodd, G. Nature 1982, 299, 352-355.

[49] Han, L.; Shi, X.; Wu, W.; Kirk, F. L.; Luo, J.; Wang, L.Y.; Mott, D.; Cousineau, L.; Lim, S. I-Im;. Lu, S.; Zhong, C. J. Sens. Actuators B. 2005, 106, 431 .

[50] Crooks, R. M.; Ricco, A. J. Acc. Chem. Res. 1998, 31, 219.

[51] Grate, J. W. Chem. Rev. 2000, 100, 2627.

[52] Davide, F.A.M.; Di Natale, C.; D’Amico. Biosens. Bioelectron. 1995, $10,203-218$.

[53] Persaud, K.C. Anal. Chem. 1992, 11, 61-67. 
[54] Shirley, S.G.; Persaud, K.C. Sernm. Neurosci.1990, 2, 59-68.

[55] Shurmer, H.V. Intern. Electric. Eng. Proc. 1990, 137, 197-204.

[56] Gardner, J.W.; Bartlett, P.N. Sens. Actuators B. 1994, 18,211-220.

[57] Schaller, E.; Bosset, J.O.; Esher F. Lebensm.-Wiss.Ul.-Technol. 1998, $31,305-316$.

[58] Mielle, P. Trends Food Sci. Technol. 1996, 7, 432-438.

[59] Demarne, V.; Grisel, A.; Sanjinks, R.; Rosenfeld, D.; Lévy, F. Sens. Actuators B. 1992, 7, 704-708.

[60] Nylander, C.; Armgarth, M.; Lundström, I. Anal. Chem. Symp. Ser. 1983, 17, 203-207.

[61] Wohltjen, H.; Dessy, R. Anal. Chem. 1979,51, 1458-1475.

[62] Ibañez, F. J.; Zamborini, F. P. ACS. Nano. 2008.2.1543-1552.

[63] Sun, Q; Selloni, A; Scoles, G. ChemPhysChem. 2005, 6, 1906 - 1910

[64] Walt, D. R. Anal. Chem. 2005, 77, 45a.

[65] Wang, L.; Luo, J.; Yin, J.; Zhang, H.; Wu, J.; Shi, X.; Crew, E.; Xu, Z.;

Rendeng, Q.; Lu, S.; Poliks, M.; Sammakia, B.; Zhong, C.-J. J. Mater. Chem. 2009, 20, 907.

[66] Joseph, Y.; Guse, B.; Vossmeyer, T.; Yasuda, A. J. Phys. Chem. C 2008, I12, 212507.

[67] Han, L.; Daniel, D. R.; Maye, M. M.; Zhong, C.-J. Anal. Chem. 2001, 73, 4441 .

[68] Wang, L.; Luo, J.; Yin, J.; Zhang, H.; Wu, J.; Shi, X.; Crew, E.; Xu, Z. 
Rendeng, Q.; Lu, S.; Poliks, M.; Sammakia, B.; Zhong, C.-J.

J. Mater. Chem. 2009, 20, 907.

[69] Zhou, H. Y.; Homer, M. L.; Shevade, A. V.; Ryan, M. A.

IEEE Sens. J. 2006, 6, 1.

[70] Hostetler, M. J.; Wingate, J. E.; Zhong, C.-J.; Harris, J. E.; Vachet, R. W.;

Clark, M. R.; Londono, J. D.; Green, S. J.;Stokes, J. J.; Wignall,

G. D. Langmuir 1998, 14, 17-30.

[71] Hudnell, H.K.; Otto, D.A.; House, D.E.; Molhave, L. Arch. Environ.

Health. 1992, 47, 31-38.

[72] Buhlmann, K.; Schlatt, B.; Cammann, K.; Shulga, A. Sens. Actuators, B. $1998,49,156-165$.

[73] Franke W.; Frechen F. B.; Giebel S. Water Sci. Technol. 2009, 59, 1721-1726.

[74] Lindinger W; Hansel A; Jordan A. Int. J. Mass Spectrom. 1998, 173, 191-241.

[75] Xu, Y.; Cheung, W.; Winder, C.L.; Goodacre, R. Anal. Bioanal. Chem. 2010, 397, 2439-2449.

[76] Penza, M.; Cassano, G.; Aversa, P.; Cusano, A.; Cutolo, A.; Giordano, M.; Nicolais, L. Nanotechnology 2005, 16, 2536-2547.

[77] Consales, M.; Campopiano, S.; Cutolo, A.; Penza, M.; Aversa, P.; Cassano, G.; Giordano, M.; Cusano, A. Sens. Actuators, B. 2006, 118, 232-242.

[78] Kiesele, H.; Wittich, M.H. E. Dräger Rev. 2000, 85, 10-13.

[79] Gardner, J.W.; Bartlett, P.N. Press: Oxford, UK, 1999; pp. 221-245.

[80] Matsuguchi, M.; Uno, T. Sens. Actuators, B. 2006, 113, 94-99. 
[81] Warneke, C.; De Gouw, J.A.; Kuster, W.C.; Goldan, P.D.; Fall, R. Environ. Sci. Technol. 2003, 37, 2494-2501.

[82] Egashira, M.; Shimizu, Y. Sens. Actuators 1993, 14, 443-446.

[83] Nanto, H.; Sokooshi, H.; Kawai, T.. Sens. Actuators 1993, 14, 715-717.

[84] Shurmer, H.V.; Gardner, J.W.; Chan, H.T. Sens. Actuators 1989, 18, 359-369.

[85] Lonergan, M.C.; Severin, E.J.; Doleman, B.J.; Beaber, S.A.; Grubbs, R.H.;

Lewis. N.S. Chem. Mater. 1996, 8, 2298-2312.

[86] Wohltjen, H.; Snow, A.W. Anal. Chem. 1998, 70, 2856-2859.

[87] Vossmeyer, T.; Guse, B.; Besnard, I.; Bauer, R.E.; Müllen, K.; Yasuda. A. $A d v$. Mater. 2002, 14, 238-242.

[88] Zhang, H.-L.; Evans, S.D.; Henderson, J.R.; Miles, R.E.; Shen, T.-H. Nanotechnology 2002, 13, 439-444.

[89] Foos, E.E.; Snow, A.W.; Twigg, M.E.; Ancona. M.G. Chem. Mater. 2002, $14,2401-2408$.

[90] Kim, Y.J.; Yang, Y.S.; Ha, S-C.; Cho, S.M.; Kim, Y.S.; Kim, H.Y.; Yang, H.; Kim, Y.T. Sens. Actuators B. 2005, 106, 189-198.

[91] Steinecker, W.H.; Rowe, M.P .; Zellers, E.T. Anal. Chem. 2007, 79, 4977-4986.

[92] Foos, E.E.; Snow, A.W.; Twigg, M.E.; Ancona, M.G. Chem. Mater. 2002, $14,2401-2408$.

[93] Ahn, H.; Chandekar, A.; Kang, B.; Sung, C.; Whitten, J. E.; Chem. Mater. 2004, 16,3274 .

[94] Im, J.; Chandekar, A.; Whitten, J.E. Langmuir 2009, 25, 4288-4292. 
[95] Castro, M.; Kumar, B.1; Feller, JF.; Haddi, Z.; Amari, A .; Bouchikhi, B.

Sens. Actuators, B 2011, 159, 213-219.

[96] Lu Y.; Meyyappan M.; Li J. J. Mater. Res. 2011, 26, 2017-2023.

[97] Wang, L.C.; Tang, K.T .; Chiu, S.W .; Yang, S.R.; Kuo, C.T .

Biosens. Bioelectron. 2011, 26, 4301-4307.

[98] Shin, K.Y. Sens. Actuators, B 2010, 147, 137-144.

[99] Raguse, B.; Barton, C.S.; Muller, K.H.; Chow, E.; Wieczorek, L . J. Phys.

Chem. C 2009, 113, 15390-15397.

[100] Rajput, NS.; Das, RR.; Mishra, VN.; Singh, KP .; Dwivedi, R. Sens. Actuators, B 2011, 155, 759-767.

[101] Fall, R.; Karl, T.; Hansel, A.; Jordan, A.; Lindinger, W. J. Geophys Res-Oc Atm. 1999, 104, 15963-15974.

[102] Abeles, B.. R. C. A. Review 1975, 36, 594.

[103] Sheng, P.; Abeles, B. Phys. Rev. Lett. 1972, 28, 34. 


\title{
CURRICULUM VITAE
}

\author{
YANG YANG \\ Department of Chemistry \\ University of Louisville \\ Louisville, KY 40292 \\ Telephone: (502) 8525982 \\ Cell phone: (502) 3458898 \\ y0yang17@louisville.edu
}

\section{Educational Background}

University of Louisville (Louisville, KY)

2009-2011

M.S. in Chemistry, December 2011

Department of Chemistry (Dr. Francis P. Zamborini)

Thesis: "Rapid functionalization of gold monolayer protected clusters for fabricating electronic noses"

University of Science and Technology of China (Hefei, China) 2005-2009

B.S. in Chemical Physics, June 2009

Department of Chemical Physics (Dr. Tianjing He)

Thesis: "Theoretical calculation of transition state in methane chlorination reaction based on density functional theory"

\section{Research Experience}

University of Louisville (Louisville, KY)

Research Assistant (Dr. Francis P. Zamborini)

Research on vapor phase place exchange reaction on gold monolayer protected clusters and vapor sensing applications. 
Undergraduate Research Assistant (Dr. Tianjing He)

Research on theoretical calculation of transition state structure in methane chlorination reaction

Dalian Institute of Chemical Physics (Dalian, China) 2008

Research Assistant for Undergraduate Research Project (Dr. Xinhe Bao)

Research on catalytic application of graphene in chemical deposition of metal clusters

\section{Publications}

Yang Yang and Francis P. Zamborini "Vapor phase thiol-place exchange reactions on films of gold monolayer-protected clusters to alter the selectivity in the chemiresistive-sensing of volatile organic compounds" manuscript in preparation, will be submitted soon.

\section{Presentations}

\section{Posters:}

Yang Yang and Francis P. Zamborini "Vapor phase thiol-place exchange reactions on thiol-coated $\mathrm{Au}$ nanoparticles: Procedure, properties and applications in altering selectivity in vapor-sensing applications" $43^{\text {rd }}$ Central Regional Meeting of American Chemical Society, Indianapolis, Indiana, -Poster (June 2011)

\section{Oral Presentations:}

Yang Yang and Francis P. Zamborini "Vapor phase thiol-place exchange reactions on film of gold monolayer protected clusters and application in altering chemiresistive sensing selectivity and building up sensor arrays for volatile organic compounds" $63^{\text {rd }}$ Southeastern Regional Meeting of American Chemical Society, Richmond, Virginia, - Oral Presentation (October 2011)

\section{Teaching Experience}

Graduate Teaching Assistant

Taught basic concepts and principles of analytical chemistry. Lectured and graded analytical laboratory courses. 
Courses Taught:

CHEM 207 - introduction to chemical analysis I - lab

CHEM 208 - introduction to chemical analysis II - lab

\section{Languages}

English, Chinese (basic)

\section{Instrumentation}

Scanning Electron Microscope (SEM), Fourier Transform Infrared Spectroscopy (FTIR), Nuclear Magnetic Resonance Spectroscopy (NMR), UV-vis Spectroscopy, E-Chem Workstation

\section{References}

Francis P. Zamborini (Ph.D.)

Department of Chemistry

University of Louisville

2320 South Brook Street

Louisville, KY 40292

Phone: (502) 8526650

Email: fpzamb01@exchange.louisville.edu

\section{Xinhe Bao (Ph.D.)}

Dalian Institute of Chemical Physics

457 Zhongshan Road

Dalian, China 116023

Phone: (86) 41184379128

Email:xhbao@dicp.ac.cn

Pawel M. Kozlowski (Ph.D.)

Department of Chemistry

University of Louisville

2320 South Brook Street

Louisville, KY 40292

Phone: (502) 8526609

Email: f.zamborini@louisville.edu 